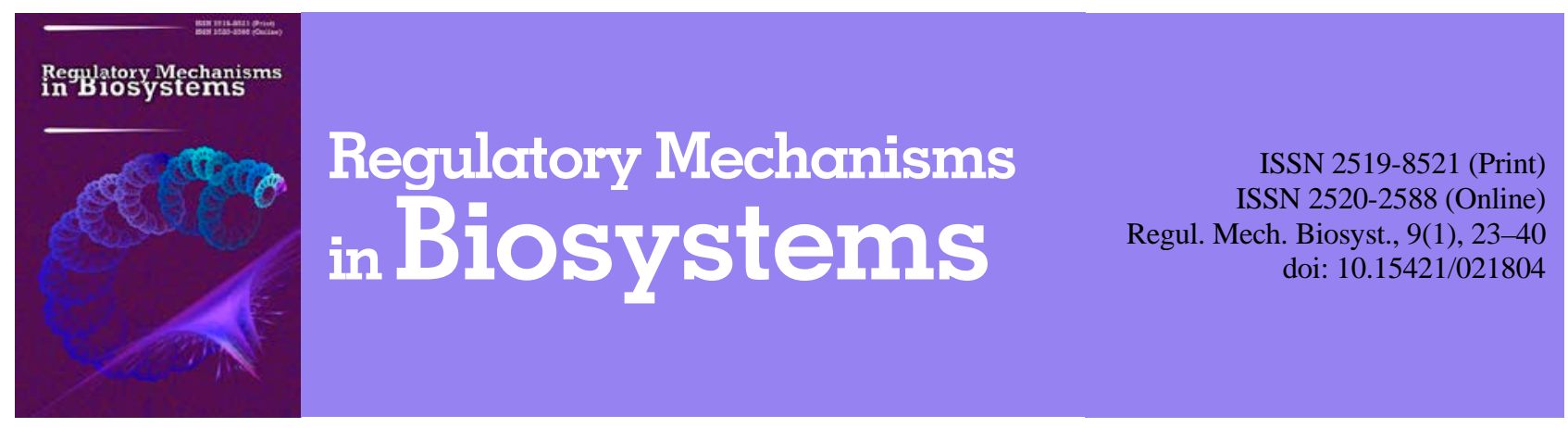

\title{
Promising new fixed combination for the treatment of diseases of the hepatobiliar system: Substantiation of pharmacotherapeutic properties and pharmaceutical quality profile
}

\author{
L. B. Bondarenko*, N. O. Gorchakova**, O. I. Golembiovska*, O. Y. Galkin******** \\ *Institute of Pharmacology and Toxicology, National Academy of Medical Sciences of Ukraine, Kyiv, Ukraine \\ **O.O. Bogomolets National Medical University, Kyiv, Ukraine \\ ***UA PRO-PHARMA LLC, Kyiv, Ukraine \\ ****National Technical University of Ukraine “Igor Sikorsky Kyiv Polytechnic Institute”, Kyiv, Ukraine
}

Article info

Received 15.01.2018

Received in revised form 24.02.2018

Accepted 27.02.2018

Institute of Pharmacology and Toxicology, National Academy Toxicology, National Academy
of Medical Sciences of Ukraine, Anton Tsedik st., 14,

Kyiv, 03057, Ukraine.

Tel. +38-044-456-42-56

O.O. Bogomolets National

Medical University,

Shevchenko blvd., 13,

Kyiv, 01601, Ukraine.

Tel. +38-044-234-40-62

UA PRO-PHARMA LLC,

Kozatska st., 120,

build. E, Kyiv, 03680, Ukraine.

Tel.: +38-044-422-50-70.

E-mail:info@pro-

pharma.com.ua

National Technical University of Ukraine "Igor Sikorsky Kyiv

Polytechnic Institute",

Peremogy av., 37,

Kyiv, 03056, Ukraine.

Tel.: +38-044-240-90-36.

E-mail:a.galkin@kpi.ua

Bondarenko, L. B., Gorchakova, N. O., Golembiovska, O. I., \& Galkin, O. Y. (2018). Promising new fixed combination for the treatment of diseases of the hepatobiliar system: Substantiation of pharmacotherapeutic properties and pharmaceutical quality profile. Regulatory Mechanisms in Biosystems, 9(1), 23-40. doi: 10.15421/021804

In this review article an analysis of literature data on the pharmacological and clinical study of a fixed combination of medicinal substances (artichoke leaf extract, ursodeoxycholic acid, taurine, and Angelica sinensis roots extract), as well a scientific substantiation of the pharmaceutical quality profile of the corresponding finished solid dosage form has been conducted. Chronic diseases of the hepatobiliary system are some of the most common human diseases and inferior only to atherosclerosis. The fact that cholecystectomy is the most common surgical operation in the abdominal organs is evidenced by the widespread distribution of the pathology of the biliary system. The fact that there is an increasing number of diagnoses of cholelithiasis in children and infants is a cause for concern. Diseases of the biliary system are closely related to violations of the functional state of the liver. Synthesis of cholesterol supplemented bile with reduced bile acid content significantly increases the risk of gallstones, as well as gallbladder cholesterol. We have substantiated that the developed preparation is a fixed combination of medicinal substances with well-researched medical applications in the treatment of dyspeptic disorders with functional disorders of the biliary system, biliary dyskinesia of the hypokinetic type, and gastritis with reflux of bile. Each of the components of the fixed combination has an important influence on the human hepatobiliary system. The effect of ursodeoxycholic acid is due to the relative replacement of lipophilic toxic bile acids, improving the secretory capacity of hepatocytes and immunoregulatory processes, which is especially important in liver and cholestatic diseases. Taurine is a synergist of ursodeoxycholic acid, since it forms biliary conjugates in the liver. The artichoke extract has choleretic, hepatoprotective and diuretic effects, while the $A$. sinensis roots extract demonstrates moderate spasmolytic and anti-inflammatory properties. We conducted a general description of active pharmaceutical ingredients and a review of biopharmaceutical tests, analyzed relevant pharmacokinetic and pharmacodynamic studies, and summarized the results of clinical efficacy and safety trials. Particular attention is paid to the results of clinical studies of the developed fixed combination. It should be noted that artichoke leaf extract, ursodeoxycholic acid, and taurine are widely used throughout the world in official medicine, at the same time, $A$. sinensis roots extract is more widely used in traditional Chinese medicine. The fixed combination has a favorable safety profile, is investigated in clinical conditions in vivo both in the form of individual components and in the form of a single drug. A fixed combination pharmaceutical profile is based on the general requirements for solid dosage forms, as well as experimentally substantiated specific indicators and research methods.

Keywords: ursodeoxycholic acid; artichoke; taurine; Angelica sinensis; clinical trials; pharmaceutical quality

\section{Introduction}

Chronic diseases of the hepatobiliary system are some of the most common human diseases and inferior only to atherosclerosis only. According to the WHO, there are more than 2 billion people in the world who suffer from liver disease, which is 100 times the prevalence of HIV infection. Over the past 20 years, there has been a clear tendency towards global increase in the number of patients with hepatobiliary diseases. There is an increase in the frequency of pathology of the hepatobiliary system in young patients, in women 4-7 times more often than in men. According to WHO experts, every 5th woman and every 10th person in Europe suffers from liver and biliary tract pathologies (Cong et al, 2011; Beuers et al., 2015).
The fact that cholecystectomy is the most common surgical operation in the abdominal organs is evidenced by the widespread distribution of the pathology of the biliary system. The increasing number of diagnoses of cholelithiasis in children and infants is a matter for concern. Diseases of the biliary system are closely related to violations of the functional state of the liver. Synthesis of cholesterol supplemented bile with reduced bile acid content significantly increases the risk of gallstones, as well as gallbladder cholesterol (Özkardeş et al., 2014; Coats et al., 2015; Tsai et al., 2015; Chhabra et al., 2016).

In recent years, there has been a search for the creation of polyfunctional action drugs. This is due to an increase in the number of patients with polymorphic pathology. At present, doctors are approached by patients who have not only one pathology, but several. According to 
modern data, the number of patients who present to their doctor with polymorbid pathology is about $80 \%$. The causes of polymorbidity are often the anatomical proximity of the affected organs, the common pathogenesis, and causal relationship. This is especially true of the hepatobiliary system, as well as diseases caused by metabolic disorders such as metabolic syndrome, diabetes mellitus, and atherosclerosis. The central place in the development of these diseases is the liver - the main organ of metabolism. Polymorbidity always involves the use of several media, which leads to polypharmacy, therefore the use of natural safe agents that have a multifunctional normalizing effect on the pathogenesis of the disease and allow reducing the use of drugs and avoiding undesirable effects of polyproagram is advisable (Kim et al., 2014; Chan et al., 2014; Uvarova el al., 2014; Pallayova et al., 2015; Tomeno el al., 2015).

As part of previous work the scientific substantiation of the safety of the pharmaceutical combination of artichoke leaf extract (200 mg), ursodeoxycholic acid (100 mg), taurine (100 mg), and Angelica sinensis roots extract $(50 \mathrm{mg}$ ) was conducted. The following preparation is designed for the treatment of dyspeptic disorders with functional disorders of the biliary system, biliary dyskinesia of the hypokinetic type, and gastritis with reflux of bile (Anokhina et al., 2014; Zvyagintseva et al., 2014; Gorchakova et al., 2017).

The aim of this work was a scientific substantiation of the pharmacodynamic and pharmacokinetic properties of the fixed combination, as well as the pharmaceutical profile of the quality of the appropriate dosage form.

\section{General characteristics of active pharmaceutical ingredients}

The proposed pharmaceutical composition is a fixed combination of drugs with well-studied medical applications.

Ursodeoxycholic acid (UDCA) is one of the native bile acids synthesized during the normal exchange of bile acids in the human body. It is an epimer of chenodesoxycholic acid and is a hydrophilic, non-cytotoxic bile acid. Ursodeoxycholic acid is the least aggressive bile acid - a natural component of human bile, its content is $1-5 \%$ of the total amount of bile acids in the human body. Applied as a medicinal product in world medicine for more than 30 years, including in Ukraine - more than 20 years (Blikhar et al., 2012; Wisher, 2012).

From the beginning, UDCA has been recommended for the dissolution of gallstones and for the treatment of reflux gastritis, and is now considered a standard for the treatment of cholestatic liver disease with an autoimmune component, such as primary biliary cirrhosis (PBC), primary sclerosing cholangitis cholangitis (PSC), etc. Currently, in the State Form of Medicines of Ukraine, it refers to the pharmacotherapeutic group of drugs used in diseases of the liver and bile ducts; the ATC code: A05A A02 (Blikhar et al., 2012; Wisher, 2012).

A small amount of UDCA is constantly contained in human bile. After oral administration, it reduces bile cholesterol saturation, slowing down the absorption of cholesterol in the small intestine and reducing cholesterol secretion into bile. Obviously, as a result of the dissipation of cholesterol and the formation of liquid crystals, partial dissolution of cholelithiasis occurs. According to modern concepts, UDCA is believed to be effective in hepatitis and cholestatic diseases due to the relative replacement of lipophilic, toxic detoxification toxic bile acids with hydrophilic cytoprotective non-toxic ursodeoxycholic acid, as well as the improvement of the secretory capacity of hepatocytes and immunoregulation processes (Blikhar et al., 2012; Wisher, 2012). It stabilizes membranes of hepatocytes and cholangiocytes, has a direct cytoprotective effect. By reducing the intake of cholesterol in the intestine and other biochemical effects, it causes hypocholesterolemic effects. It suppresses the process of cell death, caused by the action of toxic bile acids. Due to the high polarity of its molecule, UDCA is capable of producing non-toxic mixed micelles with apolar (toxic) bile acids, which reduces the ability of gastric refluxes to damage cell membranes in biliary reflux gastritis and reflux esophagitis. In addition, UDCA forms dual molecules that can be incorporated into cell membranes, stabilize them and make them insensitive to the action of cytotoxic micelles. It reduces the saturation of bile with cholesterol by inhibiting its absorption in the intestine, inhibiting the synthesis of the liver and reducing the secretion of bile; increases the solubility of cholesterol in bile, forming liquid crystals with it; reduces the lithogenic index of bile. The result is the dissolution of cholesterol gallstones (as a result of changes in the ratio of cholesterol/bile acids in bile) and preventing the formation of new concretions (as a result of a decrease in the content of bile cholesterol). In addition, ursodeoxycholic acid induces cholera, enriched with bicarbonates, which leads to an increase in the bile passages and stimulates the excretion of toxic bile acids through the intestine (Blikhar et al., 2012; Wisher, 2012).

The immune modulating effect of UDCA is due to inhibition of expression of HLA-antigens on hepatocyte and cholangiocyte membranes, normalization of the natural killer activity of lymphocytes, etc. UDCA is capable of delaying the progression of fibrosis in patients with primary biliary cirrhosis, cystic fibrosis and alcoholic steatohepatitis, reducing the risk of developing varicose veins in the esophagus (Borum et al., 1990; Cheng et al., 2012; Kotb, 2012; Buko et al., 2014).

The second component of the drug is taurine - sulfonic acid, which is formed in the body from cysteine amino acid (Blikhar et al., 2012; Wisher, 2012). Taurine is normally present in small amounts in the tissues and bile of humans and animals (Tiedemann et al., 1827; van Stijn et al., 2012).

It is synthesized in the body by enzymatic oxidation of the sulfhydryl group of cysteine with the participation of cysteine deoxygenase to cysteine sulfinic acid:

$\mathrm{HSCH}_{2} \mathrm{CH}\left(\mathrm{NH}_{2}\right) \mathrm{COOH} \rightarrow \mathrm{HO}_{2} \mathrm{SCH}_{2} \mathrm{CH}\left(\mathrm{NH}_{2}\right) \mathrm{COOH}$,

followed by decarboxylation of cysteine sulfic acid in hypotaurine: $\mathrm{HO}_{2} \mathrm{SCH}_{2} \mathrm{CH}\left(\mathrm{NH}_{2}\right) \mathrm{COOH} \rightarrow \mathrm{HO}_{2} \mathrm{SCH}_{2} \mathrm{CH}_{2} \mathrm{NH}_{2}$, and oxidation of hypotaurine in taurine (Sumizu, 1962): $\mathrm{HO}_{2} \mathrm{SCH}_{2} \mathrm{CH}_{2} \mathrm{NH}_{2} \rightarrow \mathrm{HO}_{3} \mathrm{SCH}_{2} \mathrm{CH}_{2} \mathrm{NH}_{2}$.

Taurine forms conjugates with bile acids (acylating by bile acids on the amino group), and the formed conjugates (for example, taurochole and taurodeoxycholic acids) are part of bile, and, as surfactants, contribute to the emulsion of fats in the intestine. It participates in lipid metabolism, improves energy and metabolism, stimulates healing with dystrophic diseases and processes accompanied by significant metabolic disorders of tissues (Blikhar et al., 2012; Wisher, 2012; van Stijn et al., 2012). Under conditions of systemic exposure, taurine also has hepatoprotective, cardiotonic and antihypertensive properties. It is used in cardiovascular failure, cardiac glycoside poisoning and in type 1 and type 2 diabetes mellitus (Loseva et al., 2010; Gebara et al., 2015; Gu et al., 2015; Sagara et al., 2015; Toyoda et al., 2015). Taurine is often introduced into complex medicinal products. Pharmacotherapeutic group: amino acids; the ATC code: S01X A 21. Like ursodeoxycholic acid, it has been used as a medicinal product in world medicine for more than 30 years (Blikhar et al., 2012; Wisher, 2012).

In the composition of the drug as the third pharmacologically active substance is artichoke leaves extract (Cynara cardunculus var. scolymus). A herbal remedy, a pharmacotherapeutic group: agents used in diseases of the liver and bile ducts; the ATC code: A 05A X 10. Detects choleretic, diuretic, hepatoprotective and hypolipidemic effects. Increases bile excretion, promotes excretion of nitrogen-containing substances (urea, creatinine) and toxins from the body, reduces lipid and total cholesterol in the blood, and also reduces the feeling of overflow of the stomach, relieves spasm. Pharmacological effect is caused by a complex of biologically active substances of the preparation: cynarine, chlorogenic acid, ascorbic acid, carotene, B-group vitamins, and inulin. The main active ingredient - cynarine - has a choleretic effect. The hole-kinetic effect is less. Available in artichokes ascorbic acid, carotene, vitamins $B_{1}$ and $B_{2}$, and inulin contribute to the normalization of metabolic processes. Indications for the use of an atrix extract are dyspeptic phenomena; biliary outflow, gallbladder hypokinesia, chronic hepatitis, chronic intoxication, chronic renal insufficiency, urolithiasis, urate, atherosclerosis, obesity (as part of complex therapy) (Kraft, 1997; Guarrera et al., 2005; Mootoosamy et al., 2014; Giacosa et al., 2015). Like UDCA and taurine, artichoke extract has been used as a medicinal product in world medicine for more than 30 years (Blikhar et al., 2012; Wisher, 2012). In the composition of the drug as the fourth pharmacologically active substance is Angelica sinensis extract (commonly 
known as Danggui). A herbal remedy, a pharmacotherapeutic group: other agents applied with functional disorders from the side of the gastrointestinal tract; the ATC code: A 03 A X 20.

Angelica sinensis (Oliv.) Diels is a traditional medicinal and edible plant that has long been used for tonifying, replenishing, and invigorating blood as well as relieving pain, lubricating the intestines, and treating female irregular menstruation and amenorrhea. $A$. sinensis has also been used as a health product and become increasingly popular in China, Japan, and Korea (Wei et al., 2016). The yellowish brown root of the plant is harvested in fall and is a well-known Chinese medicine used over thousands of years.

\section{Review of biopharmaceutical trials}

UDCA is a native component of the normal metabolism of bile acids in the body, whose physico-chemical properties and localization of the implementation of the physiological action predetermine the oral administration of this compound and the optimality of the capsules or tablets dosage form. Studies have shown that, in general, the degree of biliary enrichment of UDCA does not depend on the dosage form or the number of doses administered per day, but rather on the total daily dose (Blikhar et al., 2012; Wisher, 2012).

However, research on new UDCA dosage forms is ongoing. A preparation based on UDCA was developed, which is coated with a newly developed capsule film that dissolves in the stomach and releases the active substance only at $\mathrm{pH} \geq 6.5$ (Simoni et al., 1995). In 12 healthy volunteers, UDCA serum levels were measured after a single oral dose of $450 \mathrm{mg}$ of UDCA in three different dosage forms: enteric-clearing capsules, solid, non-drip gelatin capsules and conventional gelatin capsules. The drug was administered after eating. The area under the curve [AUC, mmol/ $/$ (8 hours)] after oral administration of the entericderived UDCA capsule (39.0 \pm 8.5$)$ was significantly higher than that for conventional capsules (30.5 \pm 4.9 ), both solid and non-drowning capsules $(29.3 \pm 3.4)$. In addition, the maximum serum UDCA concentration was significantly higher in the case of enteric coating capsules compared to the other two formulations, while achieving maximum UDCA concentrations in serum was observed later. These results may be explained by the assumption that the new capsule releases its contents into the intestine at the stage of the maximum alkaline $\mathrm{pH}$ in it, caused by the secretion of bile and pancreatic secretions. This improves the solubility of UDCA at alkaline $\mathrm{pH}$, thus giving it a higher concentration gradient that facilitates passive absorption.

Physico-chemical properties of UDCA have determined the specificity of biopharmaceutical studies of its dosage forms for the needs of pediatrics (Santoveña et al., 2014). Due to the very low solubility and low dose in the formula (1.5\%) UDCA for the administration of appropriate doses for infants and children was converted into a suspension with minimal use of excipients, avoiding the use of both complex supplements and those not recommended by the European Agency for the Medicines (EMA). It was obtained 2 stable (within 30-60 days) of a composition with a certain particle size.

Regarding the quantitative content of ursodeoxycholic acid in capsules, a number of clinical studies were conducted on dosage regimens. Clinical studies have shown that the administration of UDCA in the range of 10 to $15 \mathrm{mg} / \mathrm{kg} /$ day, improves liver function in chronic liver disease. With PBC, PSC, and chronic hepatitis (CH), ursodeoxycholic acid was used at doses of 250, 500 and $750 \mathrm{mg} /$ day for two months, with the treatment regimen assigned to each patient. Significant decrease in serum levels of marker enzymes was observed in all groups at doses of $250 \mathrm{mg} /$ day, which corresponds to approximately 4 $5 \mathrm{mg} / \mathrm{kg} /$ day. Two higher doses induced further improvement in the studied parameters, especially in patients with PBC, but no significant differences were found between dose levels of 500 and $750 \mathrm{mg} /$ day (Podda et al., 1989).

In a short-term, randomized, double-blind, controlled, cross-study, different UDCA doses regimens (for total dose $600 \mathrm{mg} /$ day) were studied: a course of 3 months, followed by placebo for 3 months or placebo for 3 months followed by UDCA course for 3 months and long-term UDCA courses up to 20 months (Hwang et al., 1993).
Dosage regimens of ursodeoxycholic acid up to $8.7 \mathrm{mg} / \mathrm{kg} /$ day at PBC have been studied in double-blind, multicenter trials (Battezzati et al., 1993). A comparison of three doses of ursodeoxycholic acid in the treatment of PBC was performed in another randomized study (Angulo et al., 1999). A total of 155 patients were randomized to receive low (5$7 \mathrm{mg} / \mathrm{kg} /$ day), normal (13-15 mg/kg/day) and high (23-25 mg/kg/day) UDCA doses. In this case, there were no statistically significant differences between the effects of different doses. Higher doses of UDCA (25-30 mg/kg/day) have been studied in clinical trials for patients with PSC (Harnois et al., 2001). It has been shown that the use of UDCA at a dose of $25-30 \mathrm{mg} / \mathrm{kg} /$ day may be beneficial for patients with PSC and this mode deserves further evaluation in long-term, randomized, placebo-controlled studies. A randomized, double-blind, multi-dose (300, 600, 900 mg/day) UDCA efficacy study for bile acid metabolism regimen over a period of 21 days showed a higher average UDCA dose efficiency (Hess et al., 2004). A multicentre double-blind, randomized controlled trial of UDCA prophylactic use (15 mg/kg/day) in patients after liver transplantation did not confirm the optimism of the initial report of the beneficial effect of UDCA prophylactic treatment in acute rejection after liver transplantation (Keiding et al., 1997).

Taurine is also a native component of the human body, readily soluble in water and permeable through bivarials. Since taurine implements its hepatoprotective effect together with UDCA, the choice of dosage forms in this case is limited by its physical and chemical properties. Regarding the quantitative content of taurine in capsules, a number of clinical studies were conducted on dosing regimens (Colombo et al., 1996; Heubi et al., 2002; Vettorazzi et al., 2017). In particular, taurine at a dose ( $30 \mathrm{mg} / \mathrm{kg} /$ day) was administered together with UDCA (15 mg/kg/day) during the year, resulting in an increase in its pharmacological effect in the treatment of liver disease in patients with severe pancreatic dysfunction and in poor condition nutrition (Colombo et al., 1996). Tauroursodeoxycholic acid (TUDCA) at doses of 10 $13 \mathrm{mg} / \mathrm{kg} /$ day administered for 3 months induced hepatocyte proliferation in humans (Vettorazzi et al., 2017). The high dose regimens of TUDCA (30 mg/kg/day) during enteral administration were also studied for the pediatric population (Heubi et al., 2002). However, the complex of taurine from UDCA was ineffective in preventing the development or treatment of cholestasis in newborns.

The third component of the drug is an artichoke extract. Given the herbal origin of this component and its complex chemical content, it is clear that the methods of obtaining dosage forms can play a fundamental role in the realization of its pharmacological action (BetancorFernández et al., 2003; Lupattelli et al., 2004; Ferracane et al., 2008). In particular, the study of the effect of different methods of processing artichoke herbal material significantly affects its antioxidant profile, antioxidant activity and physical characteristics (Lupattelli et al., 2004; Ferracane et al., 2008). Screening of artichoke leafy pharmaceutical preparations showed that antioxidant activity was higher in aqueous fractions than lipophilic fractions, and correlated with the total content of phenols in extracts (Betancor-Fernández et al., 2003). Regarding the quantitative content of artichoke extract in capsules, a number of clinical studies were conducted on dosage regimens (Sannia, 2010; Barrat et al., 2013). Thus, in the treatment of functional dyspeptic symptoms with oral administration of artichoke extract (15\% for chlorogenic acid, $150 \mathrm{mg}$ extract per capsule) for 60 days, the positive clinical effect, which was defined as reducing the total score of all symptoms by $50 \%$, was recorded in $38 \%$ of patients during 30 days, and $79 \%$ - within 60 days. After 60 days, in patients, the level of total cholesterol, low density lipoprotein and serum triglyceride decreased by $6-8 \%$ compared with baseline values $(\mathrm{P}<0.001)$, and the activity of transaminases and gamma-glutamyltransferase was $13-20$ units per liter $(\mathrm{P}<0.01)$ in comparison with higher initial values (Sannia, 2010). A randomized, double-blind, placebo-controlled study of low-density lipoprotein cholesterol effects in large doses of artichoke extract in patients with primary moderate hypercholesterolemia showed that doubling of the daily dose from 3 to 6 tablets for 4 weeks did not lead to additional statistically significant increase in the pharmacological effect (Barrat et al., 2013).

Radix Angelica sinensis, the dried root of $A$. sinensis (Danggui), is a herb used in Chinese medicine to enrich blood, promote blood circula- 
tion and modulate the immune system. It is also used to treat chronic constipation of the elderly and debilitated as well as menstrual disorders. Research has demonstrated that Danggui and its active ingredients, as anti-arthrosclerotic, anti-hypertensive, antioxidant anti-inflammatory agents which would limit platelet aggregation, are effective in reducing the size of cerebral infarction and improving neurological deficit scores (Hirata et al., 1997; Wu et al., 2011). Dong quai contains a chemical compound called butylidenephthalide which has antispasmodic activity in vitro and might relieve muscle cramps (Ko, 1980). Since 1970s, 165 chemical constituents, including phthalides, phenylpropanoids, terpenoids and essential oils, aromatic compounds, alkaloids, alkynes, sterols, fatty acids, and polysaccharides have been isolated or detected from the various parts of the title plant (Ma et al., 2015).

\section{Pharmacokinetic studies}

The literature does not provide data on the effects of UDCA, taurine and artichoke extract on enzymes 1A2, 2A6, 2C9, 2C19, 2D6, 2E1 of cytochrome P450 systems in human biomaterials. It has been shown that UDCA is an inducer of CYP3A, but the clinical significance of this effect is still unknown (Blikhar et al., 2012; Wisher, 2012). Investigations using quantitative polymerase chain reaction and Western blot methods of reversing the effects of ursodeoxycholic acid on proteins included in the exchange of bile acids and detoxification processes of xenobiotics in the intestine of healthy people and patients with PBC showed that UDCA induced in the gut the expression of genes of the key protein intestine pumps, as well as breast cancer resistance proteins (BCRP) and P-glycoprotein (multidrug resistance protein 1) (Dilger et al., 2012). There was no direct reverse effect on the enzymes involved in the exchange of bile acids (Wang et al., 2009). There is also data on the influence of taurine on the biosynthesis of a number of transport proteins, which are included in the cytochrome P450 2E1-dependent catabolism of xenobiotics (Miyazaki et al., 2014).

Absorption. UDCA, as a rule, is present in the form of a small fraction of the total amount of bile acids in the human body (about 5\%). After oral administration, most part of UDCA is absorbed in the small intestine and to a small extent in the colon by passive, non-ionogenic diffusion and absorption is incomplete. Subsequently, 50\% of UDCA from the portal blood when it is first passed through the liver is absorbed to conjugate with amino acids. Following a single oral administration of UDCA in a dose of $500 \mathrm{mg}$ to healthy volunteers, peak plasma concentrations were from 2.7 to $6.3 \mu \mathrm{g} / \mathrm{ml}$. The maximum concentration was reached after $60 \mathrm{~min}$, and the second peak concentration in plasma after $180 \mathrm{~min}$. Following a single oral administration of UDCA at doses of $250,500,1000$, and $2000 \mathrm{mg}$, the corresponding absorption rates were $60.3 \%$, $47.7 \%, 30.7 \%$, and $20.7 \%$. Binding to proteins in plasma is 96-98\% (Blikhar et al., 2012; Wisher, 2012).

Taurine, a sulfur-free amino acid, is a normal component of the human diet. Until recently, the pharmacokinetics of taurine in humans after oral administration had not been sufficiently studied. The study of pharmacokinetic parameters of taurine (given oral administration in the morning at $4 \mathrm{~g}$ ) was performed on 8 healthy male volunteers (mean age
27.5, range $22-45$ years). Blood samples were taken at certain intervals and the concentration of taurine in plasma was measured using a modified high-performance liquid chromatography method. Oral taurine was absorbed from the gastrointestinal tract for 1.0-2.5 hours after administration. The maximum concentration of taurine in plasma $\left(\mathrm{C}_{\max }\right)$ was $0.69 \pm 0.15 \mathrm{mmol}$ in $1.5 \pm 0.6$ hours after administration $\left(\mathrm{T}_{\max }\right)$.

The plasma half-life varied from 0.7 to $1.4 \mathrm{~h}$ (an average of $1.0 \pm$ 0.3 ), the volume of distribution - from 19.8 to 40.7 liters (an average of $30.0 \pm 7.6$ ), the ratio of clearance/bioavailability - from 14.0 to $34.4 \mathrm{l} / \mathrm{h}$ (an average of $21.1 \pm 7.8$ ), and the area under the curve for the interval $0-8$ hours (AUC) is 116.0 to $284.5 \mathrm{mg} / \mathrm{l}$ (an average of $206.3 \pm 63.9$ ) (Ghandforoush-Sattari et al., 2010). In another report, pharmacokinetic parameters were investigated in the presence of intravenous injection of $200 \mathrm{mg}$ of taurine in 6 hypertensive patients and six healthy volunteers. In this study, the half-life and the distribution of taurine were $3.85 \pm 0.05$ min and $9.6 \pm 3.2$ l, respectively. However, they monitored the concentration of taurine in blood plasma for only $20 \mathrm{~min}$, and therefore probably considered the alpha phase, which is masked by the absorption phase of taurine in the oral route of administration (De Luca et al., 2015).

Artichoke leaf extracts are traditionally used in the treatment of dyspeptic disorders and liver diseases. In order to obtain more detailed information on the absorption, metabolism and utilization of artichoke leaf extracts, two different extracts were administered to 14 healthy volunteers (Wittemer et al., 2005). Each participant in the study received doses of both extracts. The first extract - the content of caffeoylquinic acids is equivalent to $107.0 \mathrm{mg}$ of carbohydrate and the content of luteolin glycosides is equivalent to $14.4 \mathrm{mg}$ of luteolin. The second extract - the content of caffeoylquinic acids is equivalent to $153.8 \mathrm{mg}$ of coffee acid and the content of luteolin glycosides is equivalent to $35.2 \mathrm{mg}$ of luteolin. Urine and plasma tests were performed using a validated method of high-performance liquid chromatography. Peak concentrations in the blood plasma of carbohydrate, ferulic acid and isofuric acid were achieved within 1 hour. In contrast, the maximum concentrations of dihydrofuranic acid and dihydroisopropylacetate were observed only after 6-7 hours, indicating two different metabolic pathways for different caffeoylquinic acids. The peak concentration of luteolin glycosides in plasma was rapidly reached within 0.5 hours.

Regarding $A$. sinensis extract, there are some works dedicated directly to its pharmacokinetics in normal and pathological animals ( $\mathrm{Li}$ et al., 2012; Jin et al., 2017). Several studies also has been conducted to observe both drug-drug interactions and pharmacokinetics (Lo et al., 1995; Abebe et al., 2002). The main pharmacokinetic parameters of A. sinensis extract were studied and summarized by Chinese authors (Table 1) (Jin et al., 2017). A validated ultra-performance liquid chromatography-triple quadruple mass spectrometry (UPLC-TQ/MS) method was employed to quantify the a content of the main constitutes in Gui-Hong extracts (ancient and classic formula comprised of $A$. sinensis and Carthamus tinctorius L.). The results showed that Gui-Hong extracts $(0.405 \mathrm{~g} / \mathrm{ml})$ contained $144.075 \mu \mathrm{g} / \mathrm{ml}$ of hydroxysafflor yellow A (HSYA), $3.758 \mu \mathrm{g} / \mathrm{ml}$ of caffeic acid, $4.350 \mu \mathrm{g} / \mathrm{ml}$ of p-coumaric acid, $33.844 \mu \mathrm{g} / \mathrm{ml}$ of kaempferol-3-O-rutinoside, $31.647 \mu \mathrm{g} / \mathrm{ml}$ of ferulic acid, $1.583 \mu \mathrm{g} / \mathrm{ml}$ of 3-n-butylphthalide, and $3.175 \mu \mathrm{g} / \mathrm{ml}$ of ligustilide.

Table 1

Pharmacokinetic parameters of seven components of Gui-Hong extracts $(n=6)$ (by Jin et al., 2017).

\begin{tabular}{|c|c|c|c|c|c|c|}
\hline Components & $\mathrm{C}_{\max }(\mathrm{ng} / \mathrm{mL})$ & $\mathrm{T}_{\max }(\mathrm{h})$ & $\mathrm{T}_{1 / 2 \mathrm{z}}(\mathrm{h})$ & $\mathrm{MRT}_{0 \mathrm{t}}(\mathrm{h})$ & $\mathrm{AUC}_{0 \mathrm{t}}\left(\mathrm{ng} \times \mathrm{mL}^{-1} \times \mathrm{h}\right)$ & $\mathrm{AUC}_{0-\infty}\left(\mathrm{ng} \times \mathrm{mL}^{-1} \times \mathrm{h}\right)$ \\
\hline Hydroxysafflor yellow A & $25 \pm 9$ & $1.80 \pm 0.40$ & $2.60 \pm 0.30$ & $3.65 \pm 0.27$ & $116 \pm 31$ & $127 \pm 32$ \\
\hline Caffeic acid & $20 \pm 5$ & $0.75 \pm 0.00$ & $2.70 \pm 1.20$ & $2.21 \pm 0.21$ & $41 \pm 3$ & $53 \pm 11$ \\
\hline p-Coumaric acid & $750 \pm 29$ & $0.17 \pm 0.00$ & $1.04 \pm 0.37$ & $0.76 \pm 0.11$ & $430 \pm 34$ & $434 \pm 35$ \\
\hline Kaempferol-3-O-rutinoside & $17 \pm 3$ & $0.75 \pm 0.00$ & $1.00 \pm 0.53$ & $1.73 \pm 0.21$ & $31 \pm 8$ & $32 \pm 8$ \\
\hline Ferulic acid & $341 \pm 34$ & $0.17 \pm 0.00$ & $2.38 \pm 1.10$ & $1.43 \pm 0.19$ & $273 \pm 65$ & $312 \pm 77$ \\
\hline 3-n-Butylphthalide & $21 \pm 11$ & $0.17 \pm 0.00$ & $7.60 \pm 1.73$ & $2.55 \pm 0.08$ & $48 \pm 9$ & $109 \pm 25$ \\
\hline Ligustilide & $136 \pm 25$ & $0.08 \pm 0.00$ & $6.33 \pm 1.45$ & $5.69 \pm 0.76$ & $432 \pm 71$ & $463 \pm 79$ \\
\hline
\end{tabular}

Pharmacokinetic parameters of HSYA of blood stasis rats exhibited higher $\mathrm{C}_{\max }, \mathrm{T}_{1 / 2 \mathrm{z}}, \mathrm{AUC}_{0-t}, \mathrm{MRT}_{0-\mathrm{t}}$ and $\mathrm{AUC}_{0-\infty}$, and lower $\mathrm{T}_{\max }$. Among these data, $\mathrm{T}_{\text {max }}, \mathrm{AUC}_{0-\mathrm{t}}, \mathrm{AUC}_{0-\infty}$, and $\mathrm{T}_{1 / 2 \mathrm{z}}$ had a significant difference. As previously work indicated that HSYA was mainly absorbed through the small intestine, while poor blood circulation might prolong the retention time of HSYA in the small intestine, thus eventually led to

an increased bioavailability of HSYA. Additionally, slowed blood circulation may decrease the liver perfusion, which may lead to decreased hydroxylation, methylation, acetylation, and glucuronidation of the HSYA in the liver. This decreased drug metabolism may significantly increase the bioavailability of HSYA in blood stasis rats (Tian et al., 2010; Jin et al., 2016; Jin et al., 2017). 
For caffeic acid, the plasma samples from the blood stasis rats showed higher $\mathrm{C}_{\max }, \mathrm{T}_{1 / 2 z}, \mathrm{AUC}_{0-\mathrm{v}}, \mathrm{MRT}_{0-\mathrm{v}}$ and $\mathrm{AUC}_{0-\infty}$. $\mathrm{T}_{\max }$ of caffeic acid in normal and blood stasis rats was same. Meanwhile, the $\mathrm{C}_{\max }$, $\mathrm{AUC}_{0-\mathrm{v}}, \mathrm{MRT}_{0-\bullet}$, and $\mathrm{AUC}_{0-\infty}$ of caffeic acid had significant difference. Catechol-O-methyltransferase (COMT) mediated $O$-methylation plays an important role in the metabolism of caffeic acid in rat hepatocytes. Blood stasis syndromes induced by adrenaline hydrochloride or occlusion of the left anterior descending coronary artery have shown decreased COMT activity in liver plasma and the heart. The increased bioavailability of caffeic acid may partially contribute to the decreased liver and blood metabolism (Grohmann, 1987; Azuma et al., 2000; Lafay et al., 2006; Jin et al., 2017).

For $p$-coumaric acid, the model samples revealed higher $\mathrm{C}_{\max }, \mathrm{T}_{1 / 2 z}$, $\mathrm{AUC}_{0-t}, \mathrm{MRT}_{0-t}$ and $\mathrm{AUC}_{0-\infty}$. And the $\mathrm{C}_{\max }, \mathrm{AUC}_{0-\mathrm{s}}, \mathrm{MRT}_{0-\mathrm{t}}$, and AUC $_{0-\infty}$ of p-coumaric acid had a significant difference (Jin et al., 2017). Another work has demonstrated that the pharmacokinetic behavior of $p$-coumaric acid may be altered after compatibility in rats (Zeng et al., 2016). While in this study, the result indicated that the absorption of p-coumaric acid could be increased and the process of elimination was slowed in the pathological state.

As for kaempferol-3-O-rutinoside, the model samples showed higher $\mathrm{C}_{\max }, \mathrm{T}_{1 / 2 z}, \mathrm{AUC}_{0-\mathrm{t}}, \mathrm{MRT}_{0-\mathrm{t}}$, and $\mathrm{AUC}_{0-\infty}$ compared with normal rats, and the $\mathrm{T}_{1 / 2 z}, \mathrm{AUC}_{0-\mathrm{t}}, \mathrm{MRT}_{0-\mathrm{v}}$, and $\mathrm{AUC}_{0-\infty}$ of kaempferol-3-Orutinoside had significant difference. Kaempferol-3-O-rutinoside is a kind of flavonoid glycoside. A series of reasons such as high polarity, large molecules, etc., will result in the low bioavailability of kaempferol-3-O-rutinosideafter oral administration (Jin et al., 2017).

For ferulic acid with similar chemical structure of p-coumaric acid, the model rats implied higher $\mathrm{C}_{\max }, \mathrm{AUC}_{0-\mathrm{t}}$ and $\mathrm{AUC}_{0-\infty}$, and lower $\mathrm{T}_{1 / 2 \mathrm{z}}$ and $\mathrm{MRT}_{0-\mathrm{t}}$. The $\mathrm{C}_{\max }$ of ferulic acid had a significant difference. Additionally, $\mathrm{T}_{\max }$ of ferulic acid in these two groups was the same, which implied that ferulic acid absorbed more rapidly in blood stasis rats (Jin et al., 2017).

When looking at 3-n-butylphthalide, the model rats showed lower $\mathrm{T}_{1 / 2 \mathrm{z}}$ and $\mathrm{MRT}_{0-\mathrm{t}}$ and higher $\mathrm{C}_{\max }, \mathrm{AUC}_{0-t}$, and $\mathrm{AUC}_{0-\infty}$. Moreover, the $\mathrm{T}_{1 / 2 \mathrm{z}}$ and $\mathrm{AUC}_{0-\mathrm{t}}$ of 3 - $n$-butylphthalide had a significant difference. Since 3-n-butylphthalide was mainly metabolized by CYP2E1, 2C11 and $3 \mathrm{~A} 1 / 2$ in rats, which referred that model rats could change the eliminated time of 3-n-butylphthalide may be due to the changes in the enzymes (Jin et al., 2017).

Finally, for ligustilide, the model rats exhibited higher $\mathrm{C}_{\max }, \mathrm{AUC}_{0-\mathrm{t}}$ and $\mathrm{AUC}_{0-\infty}$, and lower $\mathrm{MRT}_{0-\mathrm{t}}$ and $\mathrm{T}_{1 / 2 \mathrm{z}}$. The result implied that blood stasis model rats could speed up the time and extent of absorption of ligustilide; however, there was no significant difference between normal and model rats (Jin et al., 2017).

There are also data on pharmacokinetics in animal models, for substances of known therapeutic activities (Dymowski, 2013). In a pharmacokinetic study (Luo et al., 2003) ferulic acid and paeoniflorin were detected in the serum of mice after intra-gastric administration of a combination Angelic-Paeonia root powder. The concentrations of both substances at different times were determined in serum, using HPLC. The pharmacokinetic parameters of ferulic acid in the experiment were: $\mathrm{T}_{\text {peak }}=2.606 \pm 0.586 \mathrm{~h}, \mathrm{C}_{\max }=6.372 \pm 1.510 \mathrm{mg} / \mathrm{l}, \mathrm{t}_{1 / 2(\mathrm{ka})}=1.249 \pm$ $0.365 \mathrm{~h}, \mathrm{t}_{1 / 2(\mathrm{ke})}=2.101 \pm 0.665 \mathrm{~h}, \mathrm{AUC}=41.399 \pm 11.763 \mathrm{mg} \times \mathrm{h} / \mathrm{l}, \mathrm{K}_{\mathrm{e}}$ $=0.330 \pm 0.085 \mathrm{~h}^{-1}, \mathrm{~K}_{\mathrm{a}}=0.555 \pm 0.133 \mathrm{~h}^{-1}$.

Other authors (Ru et al., 2007) observed low oral bioavailability of senkyunolide A in rats. The pharmacokinetics of senkyunolide A, of the essential oil of Rhizoma Chuanxiong (Ligusticum chuanxiong), which is commonly used for the treatment of cardiovascular diseases, was studied in rats. After intravenous administration, senkyunolide A was extensively distributed $\left(\mathrm{V}_{\mathrm{d}} / \mathrm{F}: 6.74 \pm 0.73 \mathrm{l} / \mathrm{kg}\right)$ and rapidly eliminated from the plasma $\left(\mathrm{CL} / \mathrm{F}: 7.20 \pm 0.48 \mathrm{l} / \mathrm{h} / \mathrm{kg}\right.$ and $\mathrm{t}_{1 / 2}: 0.65 \pm 0.06 \mathrm{~h}$ ). Hepatic metabolism was suggested as the major route of senkyunolide A elimination as indicated by the results of an in vitro S9 fraction study. After intraperitoneal administration, senkyunolide A exhibited dose independent pharmacokinetics. The absorption after IP administration was rapid ( $T_{\max }: 0.04 \pm 0.01 \mathrm{~h}$ ), and the bioavailability was $75 \%$. After oral administration, senkyunolide was also absorbed rapidly ( $\mathrm{T}_{\max }: 0.21$ $\pm 0.08 \mathrm{~h}$ ) however its oral bioavailability was low, about $8 \%$. Moreover as contributing factors were determined the instability in the gastrointestinal tract (accounting for $67 \%$ of the loss) and a hepatic firstpass metabolism (accounting for another 25\%). Pharmacokinetics of senkyunolide A were unaltered when the extract was administered, which suggests that components in the extract have insignificant effects on senkyunolide A pharmacokinetics.

Distribution. In healthy people, at least unconjugated, 70\% UDCA binds to plasma proteins (Rost et al., 2004). UDCA binds to one site with a protein molecule responsible for biliary bile acid binding in the ileum (the ileal bile acid-binding protein, IBABP) and increases the affinity of binding other human bile acids on the second site of IBABP. Since UDCA is one of the binding sites on IBABP, this reduces the the effect of co-operative binding, which is often observed for the major bile acids in humans. In addition, IBABP is essential for the full activation of farnesoid-X alpha receptor (FXR $\alpha$ ) bile acids, including ursodeoxycholic acid. There is no information on binding of conjugated UDCAs to plasma proteins in healthy subjects or patients with primary biliary cirrhosis. However, since the effectiveness of UDCA is due to its concentration in bile, but not in plasma, its serum levels are not an indicator of bioavailability in clinical conditions. The volume of distribution of UDCA has not been determined, however, it is believed to be small, since the compound is mainly distributed in the bile and small intestine. In bile, the concentration of UDCA reaches a peak in 13 hours (Fang et al., 2012).

Taurine can both come from outside to the body, and synthesize in the human body itself. The administered dose of taurine is rapidly absorbed and distributed over the tissues of the body. Most taurine is noted in the brain, the retina, the heart muscle, the liver and kidneys. Taurine passes through the hemathenecalfal and placental barrier (van Stijn et al., 2012).

The pharmacological activity of the artichoke extractas well as Angelica sinensis extract is due to the total effect of its components, therefore detailed pharmacokinetic studies of its distribution in human tissues have not been conducted (Blikhar et al., 2012; Wisher, 2012).

Metabolism. Approximately 50-70\% of UDCA from portal blood when it is first passed through the liver is absorbed to conjugate with amino acids (Blikhar et al., 2012; Wisher, 2012). UDCA is conjugated to glycine and taurine, and then excreted to bile and into the small intestine. In the intestine, some UDCA conjugates may be subjected to reverse processes of deconjugation and reabsorption of the ileum. UDCA conjugates may also be dehydroxyl to lithocholic acid, part of which is absorbed, sulfated in the liver and excreted from the body by the bile ducts.

In an adult, about one-quarter of bile acids are conjugated to taurine and a small portion of taurine is also converted to isotonate with the participation of either bacterial or tissue enzymes. Subsequent metabolism proceeds to the formation of sulfate, $\mathrm{CO}_{2}$, water and ammonia, the latter being converted into urea (Sturman et al., 1975).

The artichoke extract components are completely metabolized in the human body to methylated derivatives of carbohydrate (ferulous and iso-fructose acids) and to products of their hydration (dihydric and dihydrofuranic acids). With the exception of dihydrofurylic acid, all these compounds are present in the human body in the form of sulfates or glucuronides. Luteolin is completely metabolized in the human body to sulfate or glucuronide (Wittemer et al., 2005).

There are several reports on metabolism of the main bioactive ingredient of $A$. sinensis extract. Ligustilide is one of the most abundant bioactive ingredients in this plant. The study (Yan et al., 2007) reported, for the first time, the pharmacokinetics of ligustilide, administered in its pure form and in an herbal extract, in rats. After i.v. administration of pure ligustilide, it was distributed extensively $\left(V_{d}, 3.76 \pm 1.23 \mathrm{l} / \mathrm{kg}\right)$ and eliminated rapidly $\left(\mathrm{t}_{1 / 2}, 0.31 \pm 0.12 \mathrm{~h}\right)$. The i.v. clearance (CL) of ligustilide after extract administration was significantly higher than that dosed in its pure form [CL, $20.35 \pm 3.05$ versus $9.14 \pm 1.27 \mathrm{l} / \mathrm{h} / \mathrm{kg}, \mathrm{P}<$ 0.01 ; area under the curve (AUC), $0.79 \pm 0.10$ versus $1.81 \pm 0.24 \mathrm{mg} \times$ $\mathrm{h} / \mathrm{l}, \mathrm{P}<0.01$ ], suggesting significant interaction between ligustilide and components present in the extract. Dose-dependent pharmacokinetics was observed after i.p. administration, and a significantly higher dosenormalized AUC $(1.77 \pm 0.23 \mathrm{mg} \times \mathrm{h} / \mathrm{l})$ at $52 \mathrm{mg} / \mathrm{kg}$ was obtained than 
that at $26 \mathrm{mg} / \mathrm{kg}(0.93 \pm 0.07 \mathrm{mg} \times \mathrm{h} / \mathrm{l}, \mathrm{P}<0.05)$. Oral bioavailability of ligustilide was low (2.6\%), which was partly because of extensive firstpass metabolism in the liver. Seven metabolites of ligustilide were identified, and three of them were unequivocally characterized as butylidenephthalide, senkyunolide I, and senkyunolide $\mathrm{H}$. These three compounds also occurred naturally in the herb and were reported to be bioactive.

In the article (Moridani et al., 2001), evidence is presented showing that caffeic acid (CA) form plant extract when oxidized by peroxidese $/ \mathrm{H}_{2} \mathrm{O}_{2}$ or tyrosinase/ $\mathrm{O}_{2}$. Mass spectrometry analyses of the metabolites formed with peroxidase $/ \mathrm{H}_{2} \mathrm{O}_{2}$ /glutathione (GSH) revealed that mono- and bi-glutathione conjugates were formed, which formed a biglutathione conjugate only when GSH was present. In the absence of $\mathrm{GSH}$, hydroxylated products and p-quinones of CA were formed by peroxidase $/ \mathrm{H}_{2} \mathrm{O}_{2}$. NADPH also supported rat liver microsomalcatalyzed CA-glutathione conjugate formation, which was prevented by benzylimidazole, a cytochrome $\mathrm{P}_{450}$ inhibitor. Furthermore, the cytotoxicity of CA toward isolated rat hepatocytes was markedly enhanced by hydrogen peroxide or cumene hydroperoxide-supported cytochrome $\mathrm{P}_{450}$ and was prevented by benzylimidazole. Cytotoxicity was also markedly enhanced by dicumarol, an NADPH/oxidoreductase inhibittor. These results suggest that dihydroxycinnamic acids were metabolically activated by $\mathrm{P}_{450}$ peroxidase activity to form cytotoxic quinoid metabolites. Metabolism of phenolic compounds has been described in detail in several articles (Lafay et al., 2006; Zhao et al., 2010; Pei et al., 2016).

Elimination. UDCA is eliminated primarily via the faeces. In the treatment of urinary excretion, UDCA increases but remains below $1 \%$, except for severe cholestatic liver disease. In healthy volunteers receiving once orally $500 \mathrm{mg}$ of $\mathrm{C}^{14}$-spotted UDCA, $30 \%$ to $44 \%$ of the dose is excreted in the faeces during the first three days, in the form of unchanged UDCA (2-4\%), lithocholic acid (37\%), and 7-ketolithocholic acid (5\%). The half-life, determined by the use of the radioactive label, is approximately 3.5 to 5.8 days in the case of oral administration of UDCA due to the effective enterohepatic circulation of UDCA in the body. In patients with severe liver disease, renal excretion becomes one of the main ways of eliminating bile acids (Blikhar et al., 2012; Wisher, 2012).

In the case of one-time oral administration of taurine at a dose of $4 \mathrm{~g}$, its plasma concentration returns to the normal range after $8 \mathrm{~h}$ (elimination phase). The process of taurine elimination from plasma is realized in a mechanism with first-order kinetics (Ghandforoush-Sattari et al., 2010). The total content of taurine in the body is regulated by the kidneys. Taurine is one of the major urinary tract amino acids in the human body, since the ability of the kidneys to its re-absorption is low (Jacobsen et al., 1968; Chesney et al., 1985). The daily amount of taurine that is excreted in urine, although it depends on the diet, is usually in the range of 65 to $250 \mathrm{mg}$ (0.5-2.0 mmol).

Elimination of the metabolic products of the artichoke extract occurs mainly through the kidneys, with urine. The elimination profile of most products is bi-phase (Wittemer et al., 2005). Elimination of the metabolic products of the $A$. sinensis extract was reported in works we have already described (Moridani et al., 2001; Yan et al., 2007; Pei et al., 2016; Jin et al., 2017).

Pharmacokinetics in special patient groups. Pharmacokinetics and bioavailability of UDCA are independent of sex. Also, there were no clinically significant inter-racial differences in its pharmacokinetic parameters among individuals of the European and Mongolian races. Relative to the Negroid race, data is insufficient (Blikhar et al., 2012; Wisher, 2012).

Pregnant women. In the case of UDCA, taurine and artichoke extract, there are no valid and well-controlled studies in pregnant women. Since studies of the effect on animal reproductive function are not always predictive of the response in the human body, these compounds should not be used in women who are or may become pregnant. If such preparations are used during pregnancy or if the patient becomes pregnant while taking this drug, she should be aware of the potential risk to the fetus (Blikhar et al., 2012; Wisher, 2012). In the case of taurine, there are separate indications that during pregnancy it is accumulated in the tissues of the mother, and then in the perinatal period, it goes to the fetus through the placenta and the newborn through the mother's milk. It accumulates in the brain of newborns. Low levels of taurine in the maternal body lead to low levels of taurine in the fetus and to slow the growth of offspring (Naismith et al., 1987).

Breastfeeding women. It was not investigated whether orally administered UDCA, taurine and artichoke extract in maternal milk were excreted (Blikhar et al., 2012; Wisher, 2012).

Older patients (geriatrics). In the case of UDCA, taurine and artichoke extract, there are no adequate or well-controlled studies in the geriatric population (Blikhar et al., 2012; Wisher, 2012).

Children (pediatrics). In the case of UDCA, taurine and artichoke extract, there are no adequate and well-controlled studies in the pediatric population. There are only individual studies available on various aspects of the pharmacological action of these compounds in children of all ages and newborns. (Santoveña et al., 2014).

The pharmacokinetic parameters of UDCA in newborns were studied using an alpha magnetic spectrometer to quantify the concentration of sub-therapeutic doses of ${ }^{14} \mathrm{C}$-labeled UDCA (37, 120 and $370 \mathrm{~Bq}$ ) in minimal fractions $(5-25 \mathrm{ml})$ of biological liquids. However, the limited statistical sample (data from 3 newborns) and the high variability of data do not allow any definite conclusions (Santoveña et al., 2014). Since absorption in the intestine and the transition of bile acids through the portal vein of the liver are immature in young children, the pharmacokinetic parameters of UDCA established in adults may vary significantly in the pediatric population.

In the case of taurine, this amino acid is conventionally irreplaceable in humans and is now added to many baby nutrition mixtures as a precautionary measure to provide improved nutrition in premature infants and children with cystic fibrosis, as well as a positive effect on the auditory trunk of the brain caused by reactions in premature newborns (Aerts et al., 2002; Raphael et al., 2012).

\section{Pharmacodynamic studies}

When taken inside, UDCA is absorbed in the intestine due to passive diffusion, and in the ileum by means of active transport. In the liver, UDCA binds to glycine and taurine. The resulting conjugates are secreted into the bile, the acid is included in the system of hepaticintestinal circulation. With systematic administration, UDCA becomes the main bile acid in the blood serum - it accounts for about $50 \%$ of the total amount of bile acids. There is a dose-dependent increase in its share in the bile acid pool (Chernobrovy, 2013).

The enrichment of bile by UDCA makes bile more hydrophilic and less cytotoxic. UDCA modulates apical secretion in hepatocytes phosphorylation/dephosphorylation of transport proteins (activation / inactivation), which improves the excretory function of the liver. Increasing the content of UDCA in bile reduces the degree of cholangiocellular damage, portal inflammation and proliferation of the ducts. UDCA stimulates the secretion of bile acids and other organic anions (glucuronides of bilirubin, glutathione conjugates) and prevents cholestasis caused by hydrophobic bile acids. Patients with PBC and PSC treated with UDCA had less inflammatory response to the bile ducts. At the moment, the existence of three main mechanisms of action of UDCA is assumed: protection of cholangiocytes from cytotoxicity of hydrophobic bile acids; stimulation of hepatobiliary secretion; protection of hepatocytes from apoptosis caused by bile acids.

The mechanisms of UDCA cytoprotection are not fully understood. However, it is known that excessive accumulation of toxic bile acids causes apoptosis of hepatocytes. UDCA suppresses apoptosis by blocking mitochondrial dysfunction. Laboratory studies have shown that UDCA prevents apoptosis caused not only by deoxycholic acid, but also by other damaging factors (ethanol, transforming growth factor, Fas-ligand and okadaic acid). The mechanism of action of UDCA is associated with a decrease in mitochondrial depolarization with subsequent inhibition of the release of cytochrome $C$ and activation of caspases (Lazaridis et al., 2001; Pelletier et al., 2003; Venneman et al., 2006; Guarino et al., 2007; Roma et al., 2011; Portincasa et al., 2012; Guarino et al., 2013; Chernobrovy, 2013). The mechanism of inhibition of apoptosis by UDCA and tauroursodexycholic acid (TUDCA) is 
shown in Fig. 1. UDCA suppresses mitochondrial processes, namely: inhibits translocation of Bax, ROS synthesis, cytochrome C secretion, and caspase-3 activation. According to available data, UDCA may interfere with pathway of the death receptor by receptors by inhibiting caspase-3 activation. In addition, TUDCA inhibits apoptosis processes associated with EP stress by regulating intracellular levels of calcium and inhibiting calpain and activating caspase-12. In this case, it is important to note that UDCA interacts with NSAs, which leads to the dissociation of NSR/hsp90 and nuclear translocation of the complex of UDCA/NDS. Once in the nucleus, UDCA modulates the pathway of E2F-1/p53/Bax, thus preventing apoptosis. In addition, UDCA reduces cyclone D1 and Apaf-1 levels, further inhibiting the mitochondrial apoptotic cascade (Amaral et al., 2009).

In pharmacological doses UDCA significantly reduces (by 40$60 \%$ ) the saturation of bile with cholesterol by inhibiting the absorption of cholesterol in the intestine and suppressing its secretion in bile (Roma et al., 2011). UDCA reduces the toxicity of bile acids, which can damage cell membranes and cause cholestasis. This involves various mechanisms: inhibition of absorption of endogenous hydrophobic bile acids from the small intestine, choleretic effect, which causes dilution of endogenous bile acid salts in the bile ducts and thus protects hepatocytes. The recommended dose of UDCA for the treatment of cholelithiasis is $8-10 \mathrm{mg} / \mathrm{kg} / \mathrm{day}$, with larger doses there are no additional benefits. The dissolution rate is about $1 \mathrm{~mm}$ reduction in the diameter of a gallstone per month. The diameter of gallstone more than $20 \mathrm{~mm}$ negatively affects the speed of its dissolution and the overall result of litholithic therapy. Lack of dynamics or minimal reduction of concrement after 6-12 months of UDCA treatment indicate a poor dissolution prediction. The chance to reduce the diameter of large (more than $20 \mathrm{~mm}$ ) or multiple gallstones by means of litholithic therapy does not exceed $40-50 \%$ after the first year of treatment (Portincasa et al., 2012).

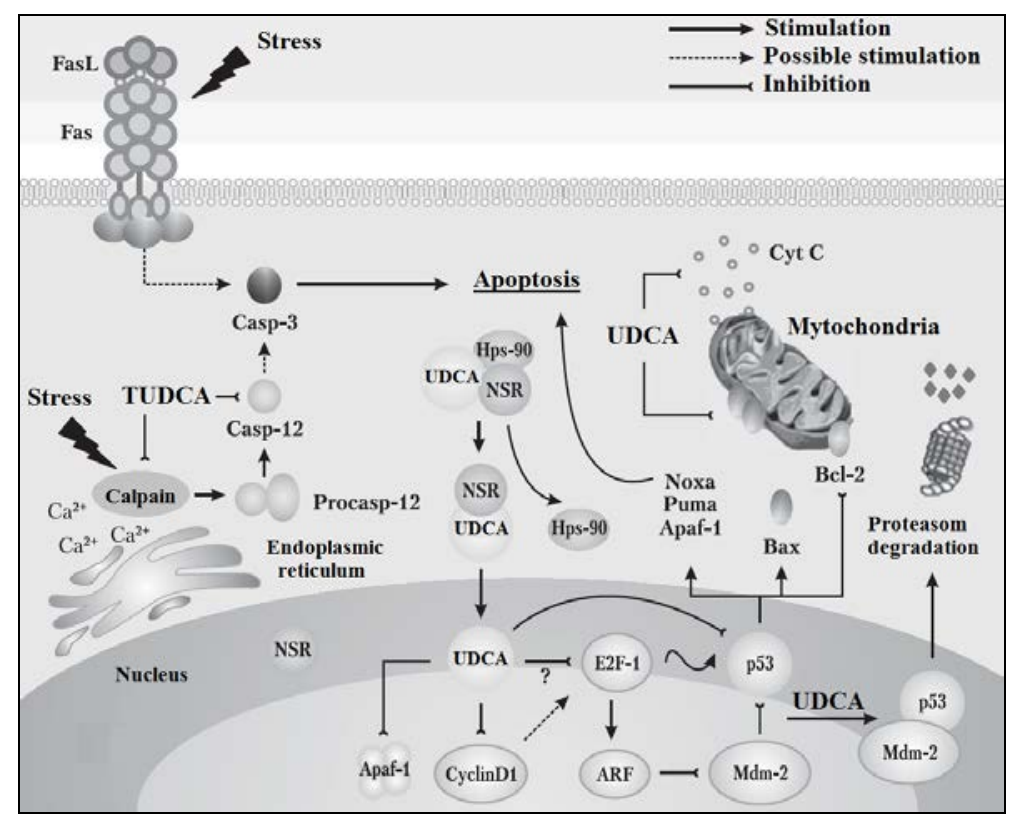

Fig. 1. Mechanisms of UDCA and TUDCA inhibition of apoptosis (by Amaral et al., 2009):

Apaf-1 - apoptotic protease activating factor 1; ARF - ARF (alternative reading frame) cell suppressor; Bax, Bcl-2 - negative (Bcl-2) and positive

(Bax) regulators of apoptosis; Casp-12, Casp-3 - caspases; Cyt C - cytochrome C; Fas - Fas cell surface death receptor; Fas L - ligand of Fasreceptor; Hsp-90 - heat shock protein; MDM-2 - mouse double minute 2 homolog; NSR - nuclear steroid receptors; Procasp-12 - procaspase

Gallbladder sludge is considered as another therapeutic target for UDCA. Its appearance can be provoked, for example, by rapid weight loss, pregnancy, parenteral nutrition, organ transplantation. A positive UDCA effect has been demonstrated in a clinical study in which patients with idiopathic acute pancreatitis and concomitant microcholletises or gallbladder sludge were taking UDCA preparations for 3 6 months. This helped to prevent relapses of cholelithiasis and pancreatitis during 44 months of observation in most patients (Lazaridis et al., 2001). UDCA also has anti-inflammatory effects. Prolonged administration of UDCA preparations significantly reduces the incidence of complications of cholelithiasis. It was found that treatment with UDCA in patients with cholelithiasis reduced the incidence of biliary pain and exacerbations of cholecystitis during a 18-year period (Tomida et al., 1999). In the first four years of receiving UDCA, relapse was less than $10 \%$, compared with $40 \%$ in the placebo group. Interestingly, this therapeutic effect did not depend on dissolution of gallstones, but was associated with a decrease in inflammatory processes in the body. Other studies (Guarino et al., 2007) indicate that UDCA treatment recovers the contractile function of the gallbladder, improves oxidative-reduction processes, eliminates oxidative stress and inflammation, and thus has a positive effect on biliary symptoms irrespective of lysis of gallstones.

The possible mechanism of reduction of inflammation is considered to be related to the antioxidant properties of UDCA, a decrease in the concentration of prostaglandin $\mathrm{E}_{2}$ and the activity of catalase. In clinical trials involving patients with severe cholecystitis and frequent biliary colic, there was no significant positive effect on the clinical course of UDCA for 100 days (Venneman et al., 2006).

The above properties of UDCA are also used in the treatment of liver parenchymal lesions. Recent experimental data indicate the expressed hepatoprotective properties of the drug in the case of the most common toxic agent - alcohol. UDCA improves functionally-morphological state of the liver. However, clinical trials of UDCA intake of 13$15 \mathrm{mg} / \mathrm{kg} /$ day for 6 months in persons with progressive alcoholic cirrhosis of the liver (Classes B and C for the Child-Pugh classification) did not show improvement in the survival of these patients (Pelletier et al., 2003). Using the modern method of diagnosis (ultrasound elastography of the liver), it was shown that patients who were taking UDCA additionally in the abstinence state had a more pronounced decrease in organ density (Mayev et al., 2010). Clinical trials of UDCA efficacy in ethanolled liver damage have not been conducted sufficiently to determine the site of this drug in the treatment of various forms of alcoholic liver disease. Perhaps this is due to the considerable heterogeneity of the clinical variants of alcoholic liver disease and the lack of generally accepted recommendations for dosage and duration of treatment. The diversity of biochemical and immunological effects of UDCA suggests that its use is possible in any clinical form of alcoholic liver disease.

An important aspect of using UDCA is the treatment of biliary reflux. The damaging effect of bile acids depends on their concentration, conjugation and $\mathrm{pH}$ of the environment. Taurine conjugates of bile acids dissolve even at $\mathrm{pH}$ 2. Thus, at low absolute values of the $\mathrm{pH}$ of 
the mucous membrane they only damage the taurine conjugates. On the contrary, at high $\mathrm{pH}$ values (for example, in the stomach coke after surgery), unconjugated bile acids have a damaging effect. The bile acids, which have detergent properties, promote the solubilization of lipids in the membranes of the surface epithelium cells. Soluble conjugated bile acids ( $\mathrm{pH} \mathrm{2-4)}$ penetrate into epithelial cells. Intracellular concentrations of bile acids can be 8 times higher than extracellular. Such excessive accumulation leads to increased permeability of cell membranes, their destruction, damage to intercellular contacts and, ultimately, loss of cells. This detrimental effect depends not only on the concentration of bile acids in reflux, but also on the duration of the period during which the mucous membrane is exposed to bile. Under the influence of pancreatic phospholipase A from biliary lecithin, lysolecithin is formed (catalyzing this reaction bile acids and trypsin). The presence of bile acids and lysolecithin in the mucous membrane of the stomach is accompanied by an increase in the inverse diffusion of hydrogen ions, as well as an increase in the release of histamine and gastrin. The negative effect of duodenogastroesophageal reflux on the mucous membrane of the esophagus has been proved. In this case, the esophagus is damaged by hydrochloric acid and pepsin, and damage to bile acids is $\mathrm{pH}$-dependent. Conjugated bile acids have a negative effect on acidic $\mathrm{pH}$ values (2-4), and unconjugated - at $\mathrm{pH} 5-8$. At alkaline $\mathrm{pH}$ values, negative effects were inherent not only on unconjugated bile acids, but also on trypsin (Sital et al., 2006).

The hydrophilicity of UDCA and the choleretic effect are important for esophagogastric protection. UDCA does not have a negative effect on the cell membrane - UDCA micelles are practically insoluble in them due to hydrophilicity. UDCA displaces toxic hydrophobic bile acids due to competitive capture of receptors. Important is the induction of cholerexis, when bile-rich bicarbonate contributes to the elimination of toxic bile acids through the intestine. The share of UDCA in gastric contents is increased to $50 \%$ with decreasing of the content of gastric and deoxycholic acids, the concentration of chenodeoxycholic acid (CDCA) does not change. In experiments and clinical studies, the cytoprotective properties of UDCA for the protection of the mucous membrane of the stomach and esophagus have been proved. This is due to UDCA in the phospholipid layer of the cell membrane, which contributes to its stabilization and increased resistance to damaging factors. In addition, the prescription of UDCA drugs reduces the subjective findings of gastric dyspepsia (Chernobrovy, 2013).

UDCA prevents the development of tumors by counteracting the stimulatory effect of other bile acids, such as deoxycholic acid (DCA). UDCA and DCA have a multidirectional effect on the epidermal growth factor (EGFR) and the expression of cyclooxygenase-2, which can play a key role in tumorigenesis in the colon. Based on convincing theoretical foundations, some clinical studies have shown that UDCA can reduce the risk of colorectal cancer, but today there are few trials with high-quality design, they are predominantly retrospective. UDCA negatively modulates the mitochondrial activation pathway by inhibiting the translocation of Bax, the formation of active forms of oxygen, the release of cytochrome $C$ and caspase- 3 (Huo et al., 2011).

Taurine is a part of the main components of bile. Conjugation is necessary to maintain the solubility of bile acids in the water environment of the intestinal contents. The presence of sulfur in tauroconjugates facilitates their ionization, increasing the detergent action and solubility, as well as reabsorption. The bile acids play an important role in preserving the function of the intestinal barrier and preventing enterobacterial invasion into tissues. In addition, bile acid tauroconjugates have choleretic effects and prevent cholestasis, unlike glycine-conjugated bile acids (Gérard, 2014). In vitro, at physiological concentrations, glycolithoholic acid is easily precipitated with calcium, unlike taurolitoholic acid. Thus, taurine is essential for increasing bile turnover, increasing bile acid production and preventing cholestasis. The introduction of taurine is likely to lower cholesterol, triglycerides, low density lipoprotein, and body weight. It reduces the amount of cholesterol in the aorta wall, the number of lipid peroxidation products, while increasing glutathione levels. It has been shown that administration of taurine inhibits cell proliferation by inhibiting the expression of mitogen-activated protein kinase (Sheybak et al., 2005).
In addition, taurine manages cellular membranes. It has membrane protection and osmoregulating effects, positively affects the phosphorlipid composition of membranes, and normalizes the electrolyte balance, holding potassium and magnesium inside the cells, and sodium from the outside. Taurine plays a very important role in the movement of calcium ions through membranes (Sheybak et al., 2005).

In particular, taurine can increase or reduce the level of calcium in the heart. It is precisely with the stabilizing effect of taurine on the membrane that its regulatory effect on the normalization of protein, carbohydrate, electrolyte metabolism, activity of a number of enzymes and hormones, energy and regenerative processes in the body, and the strengthening of the immune system are associated with its regulatory effect. Additional administration of taurine has a beneficial effect on the parameters of antioxidant defense and reduces in the experiment manifestations of diabetic neuropathy, nephropathy and retinopathy.

Taurine gives warning of a decrease in the activity of membranebound $\mathrm{Na}^{+} / \mathrm{K}^{+}$-ATPase and an excessive $\mathrm{Ca}^{2+}$ outcome. At the same time, the level of glycosylated hemoglobin and the intensity of lipid peroxidation processes in erythrocytes, which improved glucose utilization, emphasizing the potential therapeutic value of taurine in diabetes (Sheybak et al., 2005).

Another function of taurine is the preservation of euglycemia by increasing the effectiveness of binding of insulin to receptors. Use of taurine in diabetes can normalize the function of platelets and raise the level of amino acids in the blood plasma. The experiment showed that taurine improves the metabolism of glucose and lipids, reducing insulin resistance and hypercholesterolemia. Taurine warns of developing microangiopathy as a result of lowering the degree of apoptosis in endothelial cells (Sheybak et al., 2005).

It has been established that taurine can act as an antioxidant, binding active forms of oxygen. The metabolic precursor of taurine hypotaurine also has antioxidant properties. Preventive administration of taurine was reported by acute bronchiolitis induced by inhalation of $\mathrm{NO}_{2}$. Probably, in this case, taurine acts as a stabilizer for cell membranes, regulating the flow of potassium, sodium, calcium and magnesium ions (Marcinkiewicz et al., 2014). In vitro it has been shown that taurine, forming a taurochloramine, binds hypochloric acid, a strong oxidant that causes DNA damage. Taurochloramines may also play a regulatory role in inflammatory processes, inhibiting the production of interleukins 6 and 8, possibly due to a decrease in the activity of gene transcription of cytokines (Marcinkiewicz et al., 2014). The functional activity of macrophages is largely associated with the transport of taurine through a cell membrane. Treatment of macrophages with lipopolysaccharide $(0.1$ and $10 \mu \mathrm{g} / \mathrm{ml})$ leads to a $60 \%$ decrease in the transport of taurine $(\mathrm{P}<0.01)$. Transport of taurine after 24 hours did not differ from the control values in case of simultaneous treatment with lipopolysaccharide and g-interferon (150 units/ml). It was shown that inositol restores the processes of taurine transport in macrophages in conditions of its suppression (Sheybak et al., 2005). Moreover, conjugates of taurine, secondary bile acids, retinoids and some xenobiotics, due to their increased polarity after binding to taurine, become more water-soluble, which increases their clearance. This indicates the potential role of taurine in detoxification processes. The effectiveness of this amino acid in liver cirrhosis, depression and infertility in men is shown (Sheybak et al., 2005). The beneficial effects of taurine on gastric and intestinal mucosa have been described. When cystic fibrosis supplements taurine reduce the severity of steatorrhea. In Alzheimer's disease, memory decline is accompanied by a decrease in the concentration of acetylcholine.

Thus, performing numerous physiological functions in the tissues, taurine successfully modulates them in a variety of pathophysiological conditions, confirming the need for it to be found at high concentrations in the most energetically dependent cells.

The pharmacological properties of the artichoke extract are due to the effect on the patient's body of the entire complex of biologically active substances that are part of the artichoke leaf (Cynara scolymus L.). Thus, the phenolic compound of cynarine in combination with phenol acids and bioflavonoids contained in the artichoke provides choleretic, diuretic and hepatoprotective effects of the preparation. The expressive 
diuretic effect of artichoke preparations helps to increase the amount of urea that is excreted from the body and eliminate toxic substances, including so-called "middle molecules" with urine. Applying an artichoke extract provides a clear detoxifying effect, in particular, reduces the intensity of the endogenous that is, the so-called metabolic intoxication. Phenolic acids that are part of the artichoke leaf extract (coffee, chlorogenic, neochlorogenic, and caffeine) have high biological activity and cause a pronounced immunoactive action of artichoke preparations (Ben Salem et al., 2017).

Thus, the positive effect of artichoke extract and its combination with vitamin $\mathrm{E}$ (tocopherol acetate) on the lipid peroxidation indicators (LIPs) in patients with non-alcoholic steatohepatitis (NASH) is associated with chronic non-calculous cholecystitis (CNCC) against a background of secondary immunodeficiency states (SIDSs). Along with the normalization of the LIPs, namely the decrease in lipoproxidation products in the blood serum, intermediate, ie, diene conjugates (DC) and finely malone dialdehyde (MDA), there was also an improvement in the clinical and biochemical parameters that characterize the functional state of the liver and the decrease in the manifestations of SIDSs, that is, the degree of T-lymphopenia and the imbalance in the subpopulation composition of T-lymphocytes (Babak et al., 2006). In this study, it was found that when administering an artichoke extract together with vitamin E, anti-oxidant and immunomodulatory properties of this combination of drugs are implemented.

Data on the antioxidant activity of the artichoke extract, also obtained in the treatment of patients with NASH, combined with the CNCC against the background of abdominal obesity. This study demonstrated a decrease in the effect of an artichoke extract in the blood serum of products of lipid peroxidation products - MDA and DC in patients with NASH combined with CNCC in the phase of unstable remission or moderate exacerbation of the chronic inflammatory process in the gall bladder against obesity (Frolov et al., 2009). The positive effect of artichoke extract and its combination with the immune active preparation (galavit) on LIPs indices in patients with NASH combined with osteoporosis was also established.

It is known that the activity of the lipid peroxidation processes against the suppression of the activity of antioxidant defense is one of the most universal pathological mechanisms in the formation of chronic pathology of the liver and gall bladder. At the same time, phyto substances, in particular artichokes, are considered as natural antioxidant agents, established in the experiment and confirmed in clinical conditions (Ben Salem et al., 2017). Proceeding from this, it can be assumed that the use of artichoke preparations may be useful in a variety of chronic pathologies of the liver and gall bladder.

The pharmacological properties of the Angelica sinensis extract are due to the effect on the patient's body of the entire complex of biologically active substances. At the moment, the following pharmacological properties of this plant are known: effects on cardio- and cerebro-vascular systems, anti-inflammatory effect, antifibrotic action, antispasmodic activity, anti-oxidant activities, neuroprotective action, immune support and hematopoiesis (Fang et al., 2012). Proceeding from the pharmacotherapeutic profile of the complex preparation being developed, let us look in detail on the anti-inflammatory effect and antispasmodic activity.

In 1986, L. Li (Fang et al., 2012) found that sodium ferulate could regulate prostacyclin (PGI2)/thromboxane A2 (TXA2) ratio by inhibitting TXA2 activity without affecting PGI2. Ligustilide (LIG) showed a concentration-dependent anti-inflammatory effect in lipopolysaccharides (LPS) activated microglia, without causing cytotoxicity. Pretreatment with LIG at 2.5, 5, 10, and $20 \mu \mathrm{mol} / 1$ decreased LPS-induced NO production to $75.9 \%, 54.4 \%, 43.1 \%$, and $47.6 \%$; TNF- $\alpha$ content to 86.2\%, 68.3\%, 40.1\%, and 39.9\%; Interleukin- $1 \beta$ (IL-1 $\beta$ ) content to $31.5 \%, 27.7 \%, 0.6 \%$, and 0 ( $\mathrm{P}<0.01$ ); and MCP- 1 content to $84.4 \%$, $50.3 \%, 45.1 \%$, and $42.2 \%$, respectively, compared with LPS treatment alone. LIG $(10 \mu \mathrm{mol} / \mathrm{l})$ significantly inhibited LPS stimulated immunoreactivity of activated nuclear factor $\kappa \mathrm{B}(\mathrm{NF}-\kappa \mathrm{B})$, cyclooxygenase-2 (Prostaglandin-endoperoxide synthase 2), and inducible nitric oxide synthase (iNOS). LIG exerted a potent anti-inflammatory effect on microglia through inhibition of NF- $\mathrm{KB}$ pathway. The data provide direct evidence of the neuroprotective effects of LIG and the potential application of LIG for the treatment of the neuroinflammatory diseases characterized by excessive microglial activation (Wang et al, 2010; Fang et al., 2012).

Su et al. (2011), sought to determine the effects of LIG on LPSinduced inflammation in RAW 264.7 macrophages. LIG significantly suppressed the production of nitric oxide, prostaglandin E2 (PGE2), and TNF- $\alpha$. The inhibition of NO was concomitant with a decrease in the protein and mRNA levels of LPS-induced iNOS. Furthermore, activation of activator protein-1 (AP-1) and NF- $\mathrm{KB}$ in the nucleus and the cytosolic degradation of I $\mathrm{KB} \alpha$ were abrogated by LIG. LIG also inhibited the phosphorylation of IKB kinase (IKK) and mitogenactivated protein kinases (MAPKs), including p38 MAPK, extracellular signal-regulated kinase (ERK1/2) and c-Jun N-terminal kinase (JNK). The intracellular reactive oxygen species (iROS) level was also significantly decreased. These results suggest that LIG exhibits anti-inflammatory activities by blocking the activation of MAPKs/IKK and the downstream transcription factors AP-1 and NF- $\mathrm{KB}$, which may result from LIG's down-regulation of iROS production (Su et al, 2011; Fang et al., 2012). LIG significantly decreased neurological deficit score, infarct volume, and RTP801 expression, increased EPO transcription in $\mathrm{I} / \mathrm{R}$ rats, and induced a significant increase in cell viability and EPO and a decrease in LDH and RTP801 in I/R neurons. Also, LIG increased ERK phosphorylation (p-ERK) and the positive effects of LIG on p-ERK as well as cell viability and EPO could significantly be blocked by PD98059, but not LY294002 and SB203580. In addition, transfection of SH-SY5Y cells with RTP801 plasmid DNA induced a significant increase in RTP801 as well as LDH release, while LIG significantly inhibited the effects of transfection on RTP801 expression and also increased cell viability. Therefore, it suggests that LIG has a significant neuroprotecting role against I/R injury by promoting EPO transcription via a ERK signaling pathway and inhibiting RTP801 expression and has the potential to be developed into a therapeutic agent in preventing and treating ischemic disorders (Wu et al, 2011; Fang et al., 2012).

Antispasmodic activity of the $A$. sinensis extract has been studied for several decades. Methods or techniques of cell culture were used to explore the mechanism of Angelica polysaccharide (APS) inhibiting proliferation of HaCaT cells (a spontaneously transformed aneuploid immortal keratinocyte cell line from adult human skin) from the angulation of apoptosis. The effect of APS on proliferation of HaCaT cells was examined by trypan blue staining and flow cytometry. The cell growth curve showed that a dose range of 25-2500 mg/l APS had significant inhibition action on HaCaT cells in a dose dependent manner. The flow cytometry result showed a decrease in the $\mathrm{S}$ phase and G2/M phase HaCaT cells, while a phemomenal increase in the G0/G1 phase HaCaT cells was observed at $250 \mathrm{mg} / \mathrm{l}$ APS. This study suggested that APS could significantly inhibit proliferation of HaCaT cells; by impairing the mechanism of DNA synthesis in preventing HaCaT cells from entering the $S$ phase (Fang et al., 2012).

Other studies had found that intrauterin hypoxia could stimulate proliferation of neural stem cells (NSCs) of neonatal rats. The proliferation reached a peak during 6 h hypoxia; Proliferation also expressed in $9 \mathrm{~h}$, but the ability began to decline. However, the NSCs showed necrosis or apoptosis in a $12 \mathrm{~h}$ hypoxia (Chen et al, 2010). To study the effect of intrauterin hypoxia on the proliferation and differentiation of NSCs from neonatal rats and the protective role of angelica injection on NSCs under hypoxia, the authors used immunohistochemistry and an image processing system to analyze the expression of glial fibrillary acidic protein (GFAP) and neuron specific enolase (NSE). The following results were obtained from the study: (1) Expression of GFAP-positive cells in the hippocampus of neonatal rats in the hypoxia group was higher than control group; (2) Expression of NSE-positive cells was less in the hypoxia group than in the control group; (3) Expression of GFAP-positive cells in the hippocampus of neonatal rats was less in the angelica group than in the hypoxia group, whereas expression of NSE-positive cells was higher in the angelica group than in the control group. These results indicated that hypoxia could stimulate the proliferation of NSCs of neonatal rats and differen- 
tiation of NSCs into glial cells. Meanwhile, the number of neurons in hippocampus CA3 area was decreased. The ability of proliferation and differentiation of NSCs into glial cells after hypoxia was attenuated by angelica injection, which was also effective in relieving neuron decrement. Therefore, it was suggested that angelica injection has a certain protective effect on the nervous system of neonatal rats with intrauterine hypoxia (Fang et al., 2012). LIG, butylidenephthalide, and butylphthalide were found to have antispasmodic activity against rat uterine contractions and in other smooth muscle systems. The components were characterized as non-specific antispasmodics with a mechanism different from papaverine (Fang et al., 2012).

A recent study was dedicated to the hepatoprotective effect of polysaccharides from different preparations of $A$. sinensis (Hua et al., 2014). The authors report that polysaccharides are important chemical substances of $A$. sinensis. These compounds effectively treat liver diseases, show hepatoprotectivity, and contribute directly to the therapeutic effect of $A$. sinensis. However, the precise molecular mechanism of the effects of the different $A$. sinensis products polysaccharide has not been comprehensively explored. The following investigation was designed to assess the effects and possible mechanisms of polysaccharides in the different $A$. sinensis products against carbon tetrachloride-induced liver injury. Liver injury was induced by intraperitoneal injection with Carbon tetrachloride $\left(\mathrm{CCl}_{4}\right)$ in the mice. Gas chromatography-mass spectrometry (GC-MS) combined with pattern recognition approaches, namely, principal component analysis (PCA) and partial least squares-discriminant analysis (PLS$\mathrm{DA}$ ), were used to determine differentiating metabolites in plasma and liver tissue. PCA and PLS-DA score plots of the liver injury group clustered separately from that of the control, while groups treated with polysaccharides from charred $A$. sinensis (ASTP), parching A. sinensis with soil (ASTUP), parching $A$. sinensis with wine (ASJP), parching A. sinensis with Sesame Oil (ASYP) clustered closely with the control. This result indicates that the metabolic profiles of the ASTP, ASTUP, ASJP, and ASYP groups are almost similar to those of the control. Potential metabolite biomarkers (six in the liver homogenates and seven in the plasma) were identified. These biomarkers include citric acid, succinic acid,glycine, palmitelaidic acid, arachidonic acid, fumaric acid, malic acid, valine, ananine, and hexadecanoic acid. Functional pathway analysis revealed that alterations in these metabolites are associated with lipid, amino acid, and energy metabolism. Notably, ASTP exhibited a potential pharmacological effect by regulating multiple perturbed pathways to the normal state. It is likely that ASTP, ASTUP, ASJP, ASYP intervene in the metabolic process of liver injury mice by affecting the lipid and amino acid metabolism. Metabonomics is robust and promising for the identification of biomarkers and elucidation of the mechanisms of a disease, thereby highlighting its importance in drug discovery (Hua et al., 2014).

\section{Efficiency and safety studies}

Ursodeoxycholic acid has assumed a leading position in the treatment of liver and bile duct diseases during the last 30 years. The scope of UDCA use (including PBC, PSC, chronic hepatitis with cholestatic component, especially alcoholic and medicinal, cystic fibrosis, biliary tract atresia, posttransplant cholestasis, cholestasis in parenteral nutrition, intrahepatic cholestasis in pregnant women, chronic viral hepatitis - in the absence of antiviral therapy or in combination with it, NASH), has expanded in recent years and now includes the treatment of biliary reflux gastritis and reflux esophagitis, postcholecystectomy syndrome, pores antroduodenal motility ants in various diseases of the upper gastrointestinal tract, atherogenic dyslipidemia. Formal indications are lysis of cholesterol gallstones in the gall bladder and gastritis caused by bile reflux, as well as symptomatic treatment of PBC in the compensation phase. UDCA is often used in pharmacotherapy for other clinical conditions (acute and chronic hepatitis of different etiology, cholestasis) (Blikhar et al., 2012; Wisher, 2012). After the report on the dissolution of gallstones with UDCA, it was used as an alternative to surgical methods for the treatment of cholelithiasis (Roma et al., 2011; Zaretskiy et al., 2011).
The effectiveness and safety of UDCA drugs were studied in particular by Ukrainian clinicians (Zaretskiy et al., 2011). In one study, it was shown that after UDCA prescription at a dose of $15 \mathrm{mg} / \mathrm{kg} /$ day in 2-3 doses during the month, complete dissolution of concrements in patients was observed. In all observations, concrements were small from a fine dispersed suspension to $7-8 \mathrm{~mm}$ in diameter and in plural from 2 to 5 .

In another case, a clinical study was conducted on the efficacy and safety of UDCA drugs in terms of their oral administration to 70 patients. Oral litholithic therapy is by far the only truly non-invasive method for treatment of patients with gallstone disease (GSD). The benefits of such therapy include: the absence of pronounced side effects, the lack of lethality, the possibility of outpatient treatment. However, as shown by clinical studies (Zaretskiy et al., 2011), it is possible to count on the successful dissolution of gallstones only with strict selection of patients: the size of concrements should not exceed $15 \mathrm{~mm}$; it should be pure cholesterol, that is, do not give shadows on the X-ray, and should not give an "acoustic path" to the ultrasound; the gall bladder must fully maintain its function, and the bubble ducts must be passable; the gall bladder should be less than half filled with stones; the bile duct should be free from gallstones. Patients were warned that during the period of litholithic therapy they had no right to take clofibrate, estrogens, cholestiramine, antacids, because they contain compounds that bind acid bile. The most important condition for the successful dissolution of gallstones was the regularity of UDCA taking. The selection of patients also proceeded from the fact that the most favorable conditions for oral lithotripsy are in the early stages of the disease, with an uncomplicated course of GSD, rare episodes of bile duct, moderate pain syndrome.

Under supervision there were 70 patients with GSD, including 18 men and 52 women. Ultrasonography of the abdominal cavity was performed using Toshiba-33, Aloka-630 (Japan). Cholelithiasis in the examined patients was an accidental finding, the patients did not know about its existence. Their complaint was gravity in the right hypochondrium. The examination and treatment of patients was conducted from 2002 to 2007. The average age of the men was $56.4 \pm 2.9$ years, and the women $-48.7 \pm 3.4$ years. In 20 people the body weight was $60-70 \mathrm{~kg}$, in another 30 patients $-71-80 \mathrm{~kg}$, and in the rest 20 patients 81-90 kg. Excessive body mass was registered in half of the patients (35 people), among them women predominated. Most of the patients (65 people) used refined food. For all patients the functional state of the liver was evaluated on the basis of the following biochemical parameters: level of bilirubin, total protein and protein fractions, activity of alanine aminotransferase (ALT), aspartate aminotransferase (AST), gamma-glutamyltransferase (GGT), and alkaline phosphatase (ALP). Biochemical studies of blood and ultrasound were performed after 1, 3, 6,9 , and 12 months from the beginning of treatment.

In the biochemical study, some increase in the content of total bilirubin (in 8 patients) was noted, and other indicators were within the normal range. All patients had cholesterol gallstones that were usually round or oval, and were identified on the basis of appropriate criteria (Zaretskiy et al., 2011). The number of concrements in the gall bladder was from 2 to 5 . In 30 patients, the gallstones were up to $5 \mathrm{~mm}$ ib size, in another 30 - to $6-8 \mathrm{~mm}$, and in the rest 10 - to $10 \mathrm{~mm}$.

UDCA was administered in dosage of $10 \mathrm{mg} / \mathrm{kg} /$ day. The course of treatment ranged from 1 month to 1 year. UDCA was taken in capsules without chewing, before bedtime. This form of drug administration ensured its uniform release during the day. Patients were informed of the pharmacological properties of UDCA, the duration of treatment and the expected results. The diet No. 5 (Pevzner's diet) was prescribed for all patients.

One month after the beginning of the treatment, complete dissolution of gallstones was noted in 10 patients with small and single concrements, after 3 months - in 26 patients, after 6 months - in 21 patients, after 9 months - in 4 patients, and after 12 months - in 3 patients. And in 6 patients, who had gallstones of up to $10 \mathrm{~mm}$, their reduction to 2$5 \mathrm{~mm}$ was identified. In one patient developed calcinosis of gallstones was stopped with an acoustic path according to the ultrasound, and UDCA treatment. Persons with insoluble stones had an additional 2-month treatment course, after which the concrements completely dissolved. 
In general, in the world to date, only one meta-analysis of 23 randomized controlled trials (RCTs) has been conducted in which the efficacy of cholelithiasis treatment with UDCA and CDCA was studied (May et al., 1993). It covered 1949 patients who took medications at recommended doses for more than 6 months. The best result was achieved when UDCA was administered at a dose greater than $7 \mathrm{mg} / \mathrm{kg} /$ day. In 37.3\% of patients, lysis of concretions was observed.

Although gallstones consist mainly of cholesterol and should in theory dissolve, a large proportion of gastroenterologists still genuinely perceive the results of this meta-analysis and consider the actual level of gallstone dissolution to be $10 \%$. Applicants for UDCA treatment should have cholesterol (non-bilirubin) concrements without signs of measuring less than $20 \mathrm{~mm}$ in diameter and free-swallowing choledoch. The majority of clinicians consider UDCA to be the optimal dose for the treatment of cholelithiasis at rates not exceeding $10 \mathrm{mg} / \mathrm{kg} / \mathrm{day}$, since further dose increases do not significantly increase the effect. The dissolution rate is about $1 \mathrm{~mm}$ reduction in the diameter of a gallstone per month. The diameter of the concrement more than $20 \mathrm{~mm}$ negatively affects the speed of its dissolution and the overall result of litholithic therapy. Lack of dynamics or minimal reduction of concrement after 6-12 months of treatment with UDCA suggests a poor dissolution prognosis (Zaretskiy et al., 2011). The chance of reducing the diameter of large (more than $20 \mathrm{~mm}$ ) or multiple stones with the help of litholithic therapy does not exceed $40-50 \%$ after the first year of treatment (Portincasa et al., 2012).

In 2012, a meta-analysis of the hepatoprotective properties of UDCA was performed on the results of 3 RCTs with microscopic evaluation of biopsy specimens. In particular, a reduction in globular inflammation and a level of GGT in serum was observed (Wu et al., 2012).

The most pronounced therapeutic effect of UDCA is observed in patients with PBC and PSC. The performed meta-analyzes of the RCTs showed that the drug administration not only improved the patients' well-being and biochemical parameters, reduced their itching, but also influenced survival. However, a new systematic review did not confirm this (Rudic et al., 2012). The review covered 16 RCTs (1,447 patients) and did not demonstrate significant benefits of UDCA adding to standard circuits at endpoints such as death from the underlying disease, death from all causes, and the need for liver transplantation. The intensity of skin itching and fatigue were also not significantly improved. The possible cause of inconsistency with previous outcomes could be the predominance of patients with severe and terminal stages of the disease when UDCA treatment could not be effective.

A similar situation was observed with the PSC patients. A group of Greek researchers led by S. Triantos in 2011 conducted a meta-analysis of 8 placebo-controlled studies that covered the treatment outcome of 567 patients (Triantos et al., 2011). The study was completed in the last decade. The effect on the course of the disease of receiving high ( $>15 \mathrm{mg} / \mathrm{kg}$ ) doses of UDCA was studied. The drug did not contribute to reducing mortality, intensity of itching, general weakness, the risk of cholangiocarcinoma. The histological picture also did not change significantly. These data were confirmed in the following year by the meta-analysis of G. Poropat and co-authors which, despite the probable improvement in biochemical parameters, did not show a reduction in the risk of death and improvement of the histological picture (Chen et al., 2003). UDCA did not reduce the need for transplantation, did not prevent portal hypertension and encephalopathy.

A double-blind, double-simulated, randomized (1: 1), cross-linked, multi-center clinical trial was conducted in accordance with a two-stage, group-level, adaptive design in eight centers in Germany and one in the Netherlands. Two different UDCA drugs (pills and capsules) were studied in this clinical trial (Hopf et al., 2013). A total of 65 patients were recruited and randomized in the trial. The therapeutic equivalence of UDCA tablets and UDCA capsules with the effect on serum biochemical parameters was equal. Regarding safety, the total number of registered adverse events (AE) was 98. AEs were reported in 43/64 (67.2\%) patients. The overall incidence of AEs occurring during treatment with UDCA tablets was very similar to their frequency when treated with UDCA capsules: 28/62 (45.2\%) patients and 29/61 (47.5\%) patients respectively. Most AEs were digestive disorders (upper abdominal pain, bloating, abdominal distension, nausea, vomiting). No deaths were recorded during the study. However, two serious side effects were reported due to hospitalization, which were assessed as not related to UDCA therapy. One patient had a nasal septum operation and one patient had the aortic valve replaced as a result of stenosis. In general, both drugs were well tolerated, and the safety analysis showed the same UDCA safety profile for UDCA tablets and capsules. Three clinically relevant parameters were chosen in this study to assess therapeutic efficacy (ALT, AST, and GGT). In general, the maximum UDCA effect on liver function parameters was observed after 8 weeks. Clinical efficacy of UDCA capsules and tablets in patients with $\mathrm{PBC}$ was demonstrated, and confirmed by the results of a pharmacokinetics study at a daily dose of $15 \mathrm{mg} / \mathrm{kg}$. Both UDCA dosage forms were well tolerated and had similar security profiles. However, almost three times as many patients preferred to take pills (45.3\%), compared with only $15.6 \%$ of patients who preferred capsules.

According to evidence-based medicine, the hepatoprotective effect of UDCA is currently considered ambiguous, but the use of hard endpoints (death, organ transplant, etc.) may not always be able to demonstrate the potency of hepatotoxic agents. Systematic reviews of almost all authors dedicated to UDCA pharmacotherapy emphasize the lack of high-quality randomized, high-quality randomized trials without risk of systematic error, random errors, and shifting results. Consequently, it will be possible to prove or disprove the hepatoprotective properties of UDCA only when conducting such studies in the future.

Recently, a relatively new and detailed study for UDCA - biliary sludge (initial manifestation of GD) - has being conducted (Gubska, 2013). It is the possibility of prescribing UDCA for this category of patients which makes UDCA preparations particularly interesting not only for gastroenterologists, but also for doctors-physicians, family physicians, etc. Sludge is defined as a viscous suspension in the cystic bile, which includes dense structures (0.05-1.2 mm crystals) and small concrements - up to 2-3 mm in diameter. Therefore, the synonym of biliary glucose is biliary microlithiasis (presence in the cavity of the gallbladder of thickened bile, which creates a clearly defined echostructure by ultrasound, slowly moves when the patient's position changes). The clinical significance of biliary sludge has been debated, as there are numerous observations of its involuntary disappearance of the latter in a certain (18-70\%) category of patients. However, biliary sludge is rarely asymptomatic. It has been shown that in $25-70 \%$ of patients, the presence of the biliary sludge, one way or another, is accompanied by symptoms of biliary dyspepsia, pain syndrome (colic in 9-15\% of patients) and dysfunction of the sphincter of Oddi. Perhaps one of the most unfavorable and terrible complications of biliary sludge, in addition to the abovementioned attacks of biliary colic, episodes of cholecystitis, cholangitis (including purulent), the formation of gallstone disease and stenotic papillitis, is acute pancreatitis. According to various authors, it is biliary sludge that causes the formation of acute idiopathic pancreatitis in 33-90\% of patients (Gubska, 2013).

In accordance with the classification of GSD by the Russian Gastroenterologists Association (2003), biliary sludge is an early manifestation of the disease, which is noted in its first (initial) stage (Hohlacheva et al., 2016). Therefore, in most cases, in the presence of biliary sludge, a drug correction is required, for example with UDCA preparations. It is not only possible but also necessary to carry out a successful medical prophylaxis of gallstone disease. First of all, it is actual for overweight people who are eager to lose weight.

In 2007, recommendations were adopted in Germany for the diagnosis and treatment of GSD, according to which the administration of UDCA in a daily dose of not less than 500 mg for 3-6 months significantly reduces the risk of developing gallstone disease in the face of rapid decline body weight (over $1.5 \mathrm{~kg}$ per week) (Lammert et al., 2007).

UDCA preparations are interesting not only for their hepatotropic properties, but also for a large number of extrahepatic clinical effects. Relatively new indications for the use of UDCA are reflux gastritis and esophagitis due to alkaline biliary reflux. The hydrophilicity of UDCA and the choleretic effect are important for esophagogastric protection. UDCA displaces toxic hydrophobic bile acids due to competitive capture of receptors. Important is the induction of cholerexis, when bile- 
rich bicarbonate contributes to the elimination of toxic bile acids through the intestine.

It has been established that alkaline gastroesophageal reflux occurs in 5-20\% of patients with gastroesophageal reflux disease (GERD). During the pathological rejection of duodenal reflux, the destruction of the mucin barrier of the esophagus mucosa is accompanied by aggressive pancreatic enzymes (especially trypsin) and bile acids, which complicates the course of the GERD and ultimately - may be complicated by the development of the Barrett esophagus and the adenocarcinoma of the esophagus (Gubska, 2013).

Recent studies have shown that with bile reflux, the optimal dose of UDCA is $500 \mathrm{mg}$ per day ( $250 \mathrm{mg}$ in 2 doses) due to the ability of the bile acids contained in the refluxate to pass through the UDCA in a water-soluble form that to a lesser extent irritates the mucous membrane of the stomach and esophagus. The course of treatment for biliary reflux is at least two months. The share of UDCA in gastric contents is increased to $50 \%$ with decreasing of the content of gastric and deoxycholic acids, the concentration of CDCA does not change. In experiments and clinical studies, the cytoprotective properties of UDCA for the protection of the mucous membrane of the stomach and esophagus have been demonstrated (Thao et al., 2008; Chernobrovy et al., 2013). This is due to UDCA in the phospholipid layer of the cell membrane, which contributes to its stabilization and increased resistance to damaging factors. In addition, the prescription of UDCA drugs reduces the subjective findings of gastric dyspepsia.

It should be noted that extrahepatic UDCA effects are related to inflammatory bowel disease, as well as primary and secondary prevention of colorectal cancer as a result of inflammatory bowel disease. Thus, the UDCA prophylactic effect on colorectal cancer is that patients with poorly differentiated dysplasia who received UDCA for 2 years did not show deterioration, while $22.2 \%$ of patients who did not take UDCA had progression to dysplasia up to the need for colectomy. In the study of Alberts et al. (2005) the effectiveness of secondary prevention of oncogenesis in 661 patients who received UDCA for 6 months has been proved.

Clinical trials in patients with primary biliary cirrhosis indicate that the conjugate of ursodeoxycholic acid with taurine (TUDCA) has metabolic properties that can promote its long-term use as an alternative to ursodeoxycholic acid for patients with chronic cholestatic liver disease. However, direct comparison of TUDCA and UDCA in primary biliary cirrhosis has not been carried out for a long time (Larghi et al., 1997).

Finally, the effects of ursodeoxycholic and tauroursodeoxycholic acids were compared in 23 patients with PBC in a cross-linked clinical trial (Larghi et al., 1997). Both drugs were administered randomly, at a daily dose of $500 \mathrm{mg}$ for two 6-month periods separated by a 3-month washout period. The biochemical parameters of blood serum (levels of activity of enzymes reflecting the functional state of the liver and associated with cholestasis and cytolysis) consistently improved compared to baseline values, both when administered with ursodeoxycholic and with the administration of taurouxedoxycholic acid, but no significant differences were found between the pharmacological effects of these two bile acids. Both preparations were well tolerated and none of the patients complained of side effects. In the short term, tauroursodeoxycholic acid appears safe and, at least as effective as ursodeoxycholic acid, as a means of PBC treatment (Larghi et al., 1997).

Another group of researchers also showed interest in tauroursodeoxycholic acid, which, due to its high hydrophilicity, may have a significant therapeutic value in the treatment of chronic cholestatic liver disease. They conducted a study of the dependence of the pharmacological effect of TUDCA on its dose in 24 patients with PBC that were randomly assigned to receive 500, 1000, and $1500 \mathrm{mg}$ of TUDCA per day for six months. It was shown that the level of UDCA bile saturation was in the range from $15 \%$ to $48 \%$ and was not related to the dose of the drug. The biochemical parameters of blood serum (levels of activity of enzymes reflecting the functional state of the liver and related to cholestasis and cytolysis) decreased significantly after the first month of treatment with all three doses of TUDCA. There were no significant differences between the three doses at the time of interim control, but further further reduction of biochemical parameters occurred in patients with the administration of 1000 and $1500 \mathrm{mg}$ of TUDCA per day. The level of total cholesterol and cholesterol in high density lipoprotein decreased significantly in patients who received two higher doses. Diarrhea was the only side effect. The final analysis of all data obtained from a clinical study indicated that the TUDCA dose of $10 \mathrm{mg} / \mathrm{kg} /$ day is optimal for use in long-term studies in patients with primary biliary cirrhosis (Crosignani et al., 1996).

As shown by these studies, the conjugation of taurine with bile acids not only increases their hydrophilicity and solubility, but also significantly affects the solubility of cholesterol, increasing its excretion. The introduction of not only TUDCA, but also itself, of taurine, has led to reduction of serum cholesterol levels in sick people. In a clinical blind placebo-controlled study, 22 healthy male volunteers aged 18-29 were randomly assigned to two groups that received a high-fat/high cholesterol diet for three weeks to raise serum cholesterol levels. The experimental group received 6 grams of taurine every day. At the end of the trial period, the control group had significantly higher levels of total cholesterol and cholesterol in the low density lipoprotein group than in the taurine group (Mizushima et al., 1996). The ability of taurine to improve the lipid profile was also studied by other scientists (Zhang et al., 2004; de la Puerta et al., 2010).

A number of clinical trials investigated the choleretic and hypolipidemic properties of artichoke extract and its effects in patients with symptoms of dyspepsia. A randomized, double-blind, placebo-controlled, cross-sectional study involving 20 volunteers was conducted to evaluate the choleretic effect of a single administration of an artichoke extract at a dose of $1.92 \mathrm{~g}$. Monitoring of intra-ocular secretion of bile was carried out using multichannel probes starting from 30 minutes after the administration of the drug and within 4 hours after it. Increases in bile secretion were observed in both groups: with the introduction of artichoke extract and placebo. The maximum increase in bile secretion in the group with the administration of the artichoke extract was 152\% and was reached 60 minutes after taking the drug, and in the placebo group $-40 \%$ and reached in 30 minutes. Differences between the groups with artichoke extract and placebo were statistically significant 30, 60, and 90 minutes after the administration of the drug $(\mathrm{P}<0.01)$, and 120 and 150 minutes after taking the drug $(\mathrm{P}<0.05)$ (Kirchhoff et al., 1994). The results of other clinical studies (both placebo-controlled and uncontrolled) regarding the choleretic effects of artichoke extract were summarized in the review (Kraft, 1997).

The effect of artichoke extract was also investigated in several studies in patients with non-specific complaints about the gastrointestinal tract, including dyspepsia, functional disorders of the bile ducts, constipation and stomach irritation. Patients were given up to six capsules of artichoke extract daily for six weeks (during the first study) or six months (during the second study). One capsule contains $320 \mathrm{mg}$ of standardized artichoke water extract. Both studies noted improvements in clinical symptoms and decreased total cholesterol and triglycerides levels (compared with baseline values) in patients' blood serum. An analysis of the subgroup of 279 patients with at least three out of five symptoms of irritable bowel syndrome indicated a significant reduction in the severity of symptoms after taking artichoke extract (Bundy et al., 2004). The effectiveness of artichoke extract in patients with hyperlipoproteinemia was evaluated in a randomized, double-blind, placebocontrolled, multicenter study involving 143 patients with initial concentrations of total cholesterol $>7.3 \mathrm{mmol} /$. Participants received artichoke extract at a dose of $1800 \mathrm{mg}$ per day in two divided doses, or placebo for six weeks. At the end of the study, the average concentration of total cholesterol decreased in the group with the introduction of artichoke extract $18.5 \%$ and in the placebo group at $8.0 \%$ respectively $(\mathrm{P}<$ 0.0001) (Englisch et al., 2000).

Treatment with artichoke extract also led to a significant reduction in low density lipoprotein cholesterol compared to placebo $(\mathrm{P}=0.0001)$ following a randomized, double-blind, placebo-controlled study of artichoke extract (Sahebkar et al., 2017). The average baseline total cholesterol for participants in this study was low. Analysis of results in subgroups revealed hypolipidemic effects of artichoke extract. However, the number of participants included in this trial was small and insufficient. A series of three open, uncontrolled studies on the administration 
of concentrated artichoke juice (obtained from fresh leaves and flower buds) at doses of $10 \mathrm{ml}$ three times a day for 12 weeks to 84 patients with secondary hyperlipidemia (Wider et al., 2009) was also conducted. Six weeks after treatment, the concentration of total cholesterol, low density lipoprotein cholesterol and triglycerides decreased, while the high-density lipoprotein cholesterol tended to increase.

A number of clinical trials investigated treatment of gynecological and even oncological diseases (concomitant therapy) by preparations based on $A$. sinensis extract. But we focus on those studies in which the anti-spasmolytic effect was important as well as its application in gastroenterology. One study described the retrospective observation of the treatment of a group of 200 gynaecological outpatients with dysmenorrhea, irregular menstruations, aged 16-46 and treated with the product 'Concentrated Danggui Wan' and another combination of Angelica and Astragalus. One hundred and forty-eight patients were in the treatment group, 52 in the comparator group. Diseases persisted from 6 months to 12 years, average 5 years. Inclusion criteria covered three groups of symptoms, defined as following. Dysmenorrhea: premenstrual and menstrual abdominal pain affecting work and daily activities, unstable effects of antispasmodic treatment. Irregular menstrual cycle: menstrual cycle shorter than 20 days or longer than 35 days, or in 2 consecutive months the menstruation lasted for more than 7 days. Reduced menstrual flow: menstrual period less than 2 days or progressive decline of menstrual flow. The treatment group (148 cases) was treated daily with product Concentrated Danggui Wan. Each dose unit "pill” contained $0.25 \mathrm{~g}$ of the drug. Twice a day 10-20 pills were taken each time with lukewarm water. Each treatment lasted for 4 weeks, and each patient received 2-3 treatments. The control group (52 cases) took large honeybased Angelica pills daily (ingredients of the pills were Angelica and Astragalus), twice a day, $9 \mathrm{~g}$ each time. Each treatment lasted for 4 weeks, and each patient normally received 2-3 treatments. During the treatment, all other medications for dysmenorrhea and irregular menstruation were prohibited. Effects of the treatment and the side-effects were measured. Results of treatment were assessed in a 3-step scale. Significantly effective: abdominal pain is reduced after the treatment and it no longer affects daily activities and work; menstrual cycle becomes largely normal, i.e., less than 5 days early or late; the flow increases at least one third as compared to before the treatment; menstruation lasts for 5-7 days, and other symptoms have disappeared or been alleviated. Effective: abdominal pain is reduced. With the help of painkillers, a patient can remain at his/her job. Symptoms of menstrual problems have become less severe but improvements are limited as compared to the "very effective" result. Ineffective: no improvements with abdominal pain and other problems. Menstrual cycle and flow have shown no changes. Therapy in the treatment group was assessed as significantly effective in 59/148 patients (39\%), effective in 81/148 (54\%) ineffective 7/148 (4\%). Therapy in the control group was assessed as significantly effective in 27/52 patients (52\%), effective in 18/52 (34\%) ineffective in 7/52 (13\%) patients. The author states that "there have been no clear side-effects in using Concentrated Danggui Wan for treating dysmenorrhea and irregular menstruation”. In a few cases the patients developed mild nausea but the symptom quickly disappeared when the treatment was halted; there is no more detailed data (Dymowski, 2013).

The aim of another study (Dong et al., 2004) was to explore the abnormal function of platelets and the role of Angelica sinensis injection (ASI) in patients with ulcerative colitis (UC). In 39 patients with active $\mathrm{UC}, 25$ patients with remissive UC and 30 healthy people, $\alpha$-granule membrane protein (GMP-140) and thromboxane B2 (TXB2) were detected by means of enzyme-linked immunosorbent assay (ELISA), 6keto-PGF1a was detected by radioimmunoassay, platelet count (PC) and $1 \mathrm{~min}$ platelet aggregation rate (1 min PAR) were detected by blood automatic tester and platelet aggregation tester respectively, and von Willebrand factor related antigen (vWF:Ag) was detected by the means of monoclonal-ELISA. The 64 patients with UC were divided into two therapy groups. After routine treatment and Angelica sinensis injection (ASI) + routine treatment respectively for 3 weeks, all these parameters were also detected. The PC, 1 min PAR and levels of GMP-140, TXB2, and vWF:Ag in active UC were significanrly higher than those in remissive UC and normal controls ( $\mathrm{P}<0.05-0.01)$. Meanwhile, 1 min PAR and levels of GMP-140, TXB2, and vWF:Ag in remissive UC were still significantly higher than those in normal controls $(\mathrm{P}<$ 0.05). Furthermore, 6-keto-PGF1a level in active and remissive UC was remarkably lower than that in normal control $(\mathrm{P}<0.05-0.01)$. These parameters except 6-keto-PGF1a were significantly improved after the treatment in the ASI therapy group $(\mathrm{P}<0.05-0.01)$, whereas they all were little changed in the routine therapy group $(\mathrm{P}>0.05)$. The authors conclude that platelets can be significantly activated in UC, which might be related to vascular endothelium injury and imbalance between TXB2 and 6-keto-PGF1a in the blood. ASI can significantly inhibit platelet activation, relieve vascular endothelial cell injury, and improve microcirculation in UC.

The use of Angelica sinensis preparations for stopping bleeding from haemorrhoids has been reported (Gan et al., 2010). Limited, weak evidence showed that some herbal formulae, when including Radix Sanguisorbae, Radix Rehmanniae, Fructus Sophorae, Radix Angelicae Sinensis, Radix Scutellariae, etc., may alleviate some symptoms caused by haemorrhoids. These include hematochezia, congestive haemorrhoidal cushions and inflammation of perianal mucosa in the short term.

\section{Own clinical studies of the preparation}

Over the past few years, a certain experience of use of CholoplantTau capsules preparation (artichoke leaf extract $200 \mathrm{mg}$, ursodeoxycholic acid $100 \mathrm{mg}$, taurine $100 \mathrm{mg}$, and Angelica sinensis extract $50 \mathrm{mg}$ ) (UA Pro-Pharma LLC, Ukraine) in the treatment of biliary pathology has been accumulated (Anokhina et al., 2014; Zvyagintseva et al., 2014). In particular, the use of the preparation in combination therapy in patients with dysfunction of the sphincter of Oddi was investigated (Zvyagintseva et al., 2014).

Usage of this drug substances complex allows effectively restoration of the drainage function of the biliary tract, improvement of the bile drainage, reduction of the lithogenic properties of bile, which helps to prevent the formation of concrements, normalization of the motor function, biliary tract, restoration of the tone of the sphincter of Oddi, and increases the antioxidant protection the body. The clinicians examined 23 patients with Oddi's sphincter dysfunction after cholecystectomy with a disease duration of up to 2 years, aged 19-74 (mean age $54.05 \pm 3.22$ years), and evaluated the clinical efficacy of the preparation. The diagnosis was verified using clinical, laboratory and instrumental research methods. Before treatment, moderate pain and feeling of heaviness in the right hypochondrium was observed in 21 patients (91.3\%), bitterness in the mouth - in 14 (60.9\%), nausea - in 18 (78.2\%), flatulence - in 16 (69.5\%), a stroke violation - in 12 (52.1\%). The biochemical parameters of cholestasis before treatment were bilirubin $-34.6 \pm 2.7 \mu \mathrm{mol} / 1$, cholesterol $-8.3 \pm 0,6 \mathrm{mmol} / \mathrm{l}$. With ultrasound, the enlargement of the choledochus was detected in 22 (95.6\%) patients. Patients took the drug in 2 capsules 3 times a day. The efficacy of the drug was evaluated on the 21st day of treatment. After treatment, pain and severity in the right hypochondrium disappeared in all patients who had complaints (100\%); bitterness in the mouth and nausea - in 16 (69.5\%) and 20 (86.9\%), flatulence - in 14 (60.8\%) patients, normalization of the stool occurred in 11 (47.8\%) patients. In assessing the biochemical parameters in all patients, the normalization of the indicators was observed. The level of total bilirubin in the blood serum decreased 2-fold and amounted to $17.3 \pm 2.7 \mu \mathrm{mol} / \mathrm{l}$ on average, and cholesterol level was $5.8 \pm 0.3 \mathrm{mmol} / \mathrm{l}(\mathrm{P}<0.05$ compared with the corresponding indicator before treatment). According to the ultrasound, the choledoch dimensions reached norm in 19 (82.6\%) patients. The obtained results testify to the expediency of the use of the drug in the treatment of Oddi's sphincter dysfunction after cholecystectomy, since it has a pronounced therapeutic effect in this pathology, contributes to the reduction of lithogenic properties of bile, prevents the development of gallstone formation.

\section{Pharmaceutical quality profile for new preparation}

When developing the quality specification for a new drug, the results of our previous studies and the experience of other authors regar- 
ding the analytical standardization of UDCA based drugs and phytopharmaceutical products (Dhami et al., 2015; Galkin et al., 2011; Galkin et al., 2013; Gontova et al. 2016; Lutsenko et al., 2017; Ramaekers et al., 2017), as well as regulatory requirements (European Pharmacopoeia, Ph. Eur. 9th Edition) were used.

The specification for the preparation has been developed in accordance with the requirements of the "Note for Guidance Specifications: Test Procedures and Acceptance Criteria for New Drug Substances and New Drug Products: Chemical Substances" (CPRM/ICH/ 367/96) and the manufacturer's specifications for the UDCA, taurine and artichoke extract.

Identification test. This specification test is proposed as additional to the determination of the quantitative content of the main active substances. Identification is carried out in accordance with the following methods.

The identification of the artichoke extract is carried out by the determination of hydroxycholic acids, which are the main active ingredients of dry extract of artichoke leaves (Cynara scolymus L.). This is done by adsorption spectrophotometry in the ultraviolet region, according to the requirements of Ph. Eur., 2.2.25, in assay test. The ultraviolet absorption spectrum of the test solution in the region from $250 \mathrm{~nm}$ to $400 \mathrm{~nm}$ should have a maximum absorption at the wavelength $327 \pm 2 \mathrm{~nm}$ and a shoulder in the region $300 \pm 2 \mathrm{~nm}$, since this spectrum is characteristic of the chlorogenic acid, which is the dominant hydroxychric acid in dry extract of artichoke leaves.

The identification of the ursodeoxycholic acid is carried out by liquid chromatography, according to the requirements of Ph. Eur., 2.2.29, at the same time as assay test. The retention time of the main peak of the ursodeoxycholic acid obtained on the chromatogram of the test solution was compared. It should correspond to the time of holding the peak of the ursodeoxycholic acid on the comparison solution chromatogram.

The identification of taurine is carried out by thin-layer chromatography according to the requirements of Ph. Eur., 2.2.27, simultaneously with the test "Impurities of taurine". On the chromatogram of the solution tested, the taurine spot by location, form and color must correspond to the spot of the taurine of the comparison solution.

The identification of polyphenolic compounds to the A. sinensis extract is carried out by qualitative reaction with phosphoricmolybdenum-tungsten reagent $\mathrm{P}$ (the appearance of a blue color).

Test "Uniformity of mass". No more than 2 individual masses deviate from the average mass by more than $7.5 \%$, and none of them will deviate by more than $15.0 \%$. This regulation is proposed on the basis of experimental data.

Test "Related impurities". Requirements for impurities are set according to "Note for Guidance on Impurities in New Drug Products" (CPMP/ICH/2738/99).

Related impurities of ursodeoxycholic acid. Impurity A (xenodeoxycholic acid) and impurity C (lithocholic acid) are specific impurities of the ursodeoxycholic acid. The tests are carried out by the HPLC method (impurity A, unidentified impurities, amount of impurities) in accordance with the requirements of Ph. Eur., 2.2.29, and by TLC (impurity C) according to the requirements of Ph. Eur., 2.2.27. On the chromatogram of the solution to be tested, the peak area of the impurity A (xenodeoxycholic acid) should not exceed 10 times the area of the main peak on the chromatogram of the comparison solution (1.0\%); the area of any other impurity should not exceed the area of the main peak on the chromatogram of the comparison solution (0.1\%). The sum of the areas of the peaks of all impurities should not exceed 15 areas of the main peak on the chromatogram of the comparison solution (1.5\%). An impurity C (lithoacid acid) can be detected by the HPLC method, but in the determination of other contaminant impurity of ursodeoxycholic acid, the lithoholic acid has a retention time of more than 90 minutes on the chromatogram.

Thus, the determination of the impurity is proposed to be carried out by the TLC to the requirements of Ph. Eur., 2.2.27, according to the method of the corresponding monograph on the ursodeoxycholic acid on a silica gel plate in a mobile phase ice acetic acid : acetone : methylene chloride $(1: 30: 60)$ after treatment with a solution of phosphoric molybdic acid in a mixture of sulfuric acid and acetic acetic acid and heating. Stains of vaginal acids acquire a blue color. In this case, the stain of the impurity $\mathrm{C}$ with the intensity and size is not more intense than the acid stain of the ursodeoxycholic acid on the comparison solution (less than $0.1 \%$ ).

Related impurities of taurine. The determination is carried out by the TLC according to the requirements of Ph. Eur., 2.2.27, on Silicagel 60 F 254 plate in the mobile phase butanol : ice acetic acid : water (60 : $20: 20$ ) after treatment with ninhydrin solution and heating. Solutions of amino acids, polypeptides, peptones and primary amines when heated with ninhydrin (1,2,3-indanthion) acquire a blue or violet color. In this case, no stain of the impurity should exceed the size and intensity of the staining of the taurine spot on the comparison solution (less than 0.5\%).

Dissolution test. Tests are conducted in six units in accordance with the requirements of the Ph. Eur., 2.9.3, with the use of a blade device. The amount of ursodeoxycholic acid, which has been transferred to the solution, is determined by HPLC according to the requirements of Ph. Eur., 2.2.29. For the preparation, it is proposed to use one study point for the release of active substances, since it relates to a normal release dosage forms with normal release containing rapidly soluble active substances. The $\mathrm{pH}$ of the dissolution medium (synthetic gastric juice, $\mathrm{pH}$ 8.0, without pancreatin) given in the United States Pharmacopeia monograph for tablets of ursodeoxycholic acid is not suitable for its determination in the presence of other active substances and concomitant substances (the effect of the physicochemical properties of the substance and the auxiliary substances) - there is no peak of the ursodeoxycholic acid on chromatogram of the test solution. The same is observed for the dissolution medium - the synthetic intestinal juice, $\mathrm{pH}$ 6.8, without pancreatin. However, taurine is reliably determined in these conditions.

In order to optimize the technique of dissolution test and simultaneously determine the ursodeoxycholic acid and taurine in the preparation as a dissolving medium, it is proposed to use water $\mathrm{P}$. In this case, the ursodeoxycholic acid and taurine that have been transferred to the solution are reliably determined, which is confirmed by the validation data and experimental data obtained in the step pharmaceutical development. In this way, the tests are carried out in water for 45 minutes. The drug can withstand the test if the amount of ursodeoxycholic acid and taurine that has passed into the solution in $\mathbf{4 5}$ minutes is at least $85 \%(\mathrm{Q}+5 \%)$ of the nominal content. This regulation is proposed on the basis of experimental data and Ph. Eur., 2.9.3, for solid dosage forms with traditional release.

Disintegration test. The time of full disintegration in water (disc method) should be no more than 30 minutes. This regulation is proposed on the basis of experimental data (Ph. Eur., 2.9.1, test A).

Test "Uniformity of dose units". The determination is made by the calculation and weighting method for taurine, since the content of this component is greater than $25 \mathrm{mg}$ and is more than $25 \%$ of the weight of the dosage form. The determination is carried out by a direct method for the artichoke extract and the ursodeoxycholic acid, since the drug substances content is less than $25 \%$ of the total weight of the medicinal product. Calculate the acceptance value AV using the reference number $\mathrm{M}$ for case 1 . The $\mathrm{AV}$ for the first 10 units is less than or equal to $\mathrm{L} 1=15$, or the final AV calculated from 30 units is less than or equal to L1 = 15 and no individual contents in the dosage unit is not less than $0.75 \mathrm{M}$ and not more than $1.25 \mathrm{M}$. The result must meet the requirements of the Ph. Eur., 2.9.40. This parameter is controlled during the release of the series and is not critical for the stability studies.

Microbiological purity test. In accordance with the requirements of the general article Ph. Eur., 5.1.4, the eligibility criteria for the microbiological purity of the finished non-sterile administered medicinal products for oral use are included in the specification. Tests are conducted in accordance with the requirements of Ph. Eur., 2.6.12, 2.6.13. The following conditions were established: the total number of aerobic microorganisms (TAMS) $\leq 10^{3} \mathrm{CFU} / \mathrm{g}$, the total number of yeast and mold (TYMS) $\leq 10^{2} \mathrm{CFU} / \mathrm{g}$, the absence of Escherichia coli in $1 \mathrm{~g}$.

Assay test. It is proposed to set the limit of quantitative content \pm $5 \%$ to the declared quantity of active substances during release and \pm $10 \%$ during the storage period. The quantitative content of the ursodeoxycholic acid is determined by HPLC according to the requirements of 
Ph. Eur., 2.2.29, based on the average weight of the dosage form with refractometric detection on a column filled with $5 \mu \mathrm{m}$ octadecyl silicagel, $4.6 \times 150 \mathrm{~mm}$. The content of the ursodeoxycholic acid should be $95-105 \%$ of the normal amount at release and from $90 \%$ to $110 \%$ up to the shelf life. This regulation is proposed on the basis of experimental data and requirements of $\mathrm{Ph}$. Eur.

The quantity of artichoke extract is determined by the content of the hydroxycholic acid, based on the average weight of the dosage form, by the absorption spectrophotometry method in the UV region at $327 \pm$ $2 \mathrm{~nm}$, using the optical absorption index of chlorogenic acid. The content of hydroxycinnamic acids should be not less than $5.0 \mathrm{mg}$, in terms of chlorogenic acid on average weight. This regulation is proposed on the basis of experimental data (Ph. Eur., 2.2.25).

The quantitative content of taurine is determined by the acid-base titration method. Taurin, due to the amphoteric nature, can not directly titrate with alkaline solution. Titration is possible if the amino group is blocked by the action of formaldehyde. The formed compound can be titrated alkalimetrically with a phenolphthalein indicator. The content of taurine should be $95-105 \%$ of the normal amount at release and from $90 \%$ to $110 \%$ up to the shelf life.

\section{Conclusions}

An overview of our own data and data from the literature on the pharmacological and clinical study of a fixed combination of medicinal substances (artichoke leaf extract $200 \mathrm{mg}$, ursodeoxycholic acid 100 $\mathrm{mg}$, taurine $100 \mathrm{mg}$, and Angelica sinensis roots extract $50 \mathrm{mg}$ ), as well a scientific substantiation of the pharmaceutical quality profile of the corresponding finished solid dosage form has been conducted. It is substantiated that this drug is a fixed combination of medicinal substances with well-researched medical applications in the treatment of dyspeptic disorders with functional disorders of the biliary system, biliary dyskinesia of the hypokinetic type, and gastritis with reflux of bile. Each of the components of the fixed combination has an important influence on the human hepatobiliary system. The effect of ursodeoxycholic acid is due to the relative replacement of lipophilic toxic bile acids, improving the secretory capacity of hepatocytes and immunoregulatory processes, which is especially important in liver and cholestatic diseases. Taurine is a synergist of ursodeoxycholic acid, since it forms biliary conjugates in the liver. The artichoke extract has choleretic, hepatoprotective and diuretic effects, while the $A$. sinensis roots extract demonstrates moderate spasmolytic and anti-inflammatory properties. The fixed combination has a favorable safety profile, has been investigated in clinical conditions in vivo both in the form of individual components and in the form of a single drug. A fixed combination pharmaceutical profile is based on the general requirements for solid dosage forms, as well as experimentally substantiated specific indicators and research methods.

\section{References}

Abebe, W. (2002). Herbal medication: Potential for adverse interactions with analgesic drugs. Journal of Clinical Pharmacy and Therapeutics, 27, 391-401.

Aerts, L., \& Van Assche, F. A. (2002). Taurine and taurine-deficiency in the perinatal period. The Journal of Perinatal Medicine, 30(4), 281-286.

Alberts, D. S., Martínez, M. E., Hess, L. M., Einspahr, J. G., Green, S. B., Bhattacharyya, A. K., Guillen, J., Krutzsch, M., Batta, A. K., Salen, G., Fales, L., Koonce, K., Parish, D., Clouser, M., Roe, D., \& Lance, P. (2005). Phase III trial of ursodeoxycholic acid to prevent colorectal adenoma recurrence. Journal of the National Cancer Institute, 97(11), 846-853.

Amaral, J. D., Viana, R. J. S., Ramalho, R. M., Steer, C. J., \& Rodrigues, C. M. P. (2009). Bile acids: Regulation of apoptosis by ursodeoxycholic acid. Journal of Lipid Research, 50(9), 1721-1734.

Angulo, P., Dickson, E. R., Themeau, T. M., Jorgensen, R. A., Smith, C., DeSotel, C. K., Lange, S. M., Anderson, M. L., Mahoney, D. W., \& Lindor, K. D. (1999). Comparison of three doses of ursodeoxycholic acid in the treatment of primary biliary cirhosis: A randomized trial. Journal of Hepatology, 30(5), 830-835.

Anokhina, G. A., Kharchenko, V. V., Opanasiuk, N. D., Lopukh, I. Y., \& Yakubovska, I. O. (2014). Some aspects of the use of preparations of ursodeoxycholic acid, combined with plant extracts, in the treatment of the diseases of the hepatobiliary system. Modem Gastroenterology, 1, 49-54 (in Russian).
Azuma, K., Ippoushi, K., Nakayama, M., Ito, H., Higashio, H., \& Terao, J. (2000). Absorption of chlorogenic acid and caffeic acid in rats after oral administration. Journal of Agricultural and Food Chemistry, 48, 5496-5500.

Babak, O. Y., Frolov, V. M., \& Fadeenko, U. D. (2006). Influence of the combination of articholes and vitamin $\mathrm{E}$ on the state of lipid peroxidation in patients with non-alcoholic steatohepatitis, combined with chronic noncalculous cholecystitis, against secondary immunodeficiency states. Problems of Ecological and Medical Genetics and Clinical Immunology, 6(75), 111-118 (in Ukrainian).

Barrat, E., Zaï, Y., Sirvent, P., Chauveau, P., Maudet, C., Housez, B., Derbord, E., Lescuyer, J. F., Bard, J. M., Cazaubiel, M., \& Peltier, S. L. (2013). Effect on LDL-cholesterol of a large dose of a dietary supplement with plant extracts in subjects with untreated moderate hypercholesterolaemia: A randomised, double-blind, placebo-controlled study. European Joumal of Nutrition, 52(8), 1843-1852.

Battezzati, P. M., Podda, M., Bianchi, F. B., Naccarato, R., Orlandi, F., Surrenti, C., Pagliaro, L., \& Manenti, F. (1993). Ursodeoxycholic acid for symptomatic primary biliary cirrhosis. Preliminary analysis of a double-blind multicenter trial. Italian Multicenter Group for the Study of UDCA in PBC. Journal of Hepatology, 17(3), 332-338.

Ben Salem, M., Ben Abdallah Kolsi, R., Dhouibi, R., Ksouda, K., Charfi, S., Yaich, M., Hammami, S., Sahnoun, Z., Mounir Zeghal, K., Jamoussi, K., \& Affes, H. (2017). Protective effects of Cynara scolymus leaves extract on metabolic disorders and oxidative stress in alloxan-diabetic rats. BMC Complementary and Alternative Medicine, 17, 328.

Betancor-Femández, A., Pérez-Gálvez, A., Sies, H., \& Stahl, W. (2003). Screening pharmaceutical preparations containing extracts of turmeric rhizome, artichoke leaf, devil's claw root and garlic or salmon oil for antioxidant capacity. Journal of Pharmacy and Pharmacology, 55(7), 981-986.

Beuers, U., Trauner, M., Jansen, P., \& Poupon, R. (2015). New paradigms in the treatment of hepatic cholestasis: From UDCA to FXR, PXR and beyond. Journal of Hepatology, 62(1), S25-S37.

Blikhar, V. I., Chumak, V. T., Maltsev, V. I., Morozov, A. M., Parii, V. D., Stepanenko, A. V., \& Dumenko, T. M. (2012). State form of medicines. 4th ed. State Expert Center of Ukraine.

Borum, M., \& Fromm, D. H. (1990). Expression of HLA molecules on hepatocytes: Does ursodeoxycholic acid prevent immunologic injurY? Gastroenterology, 99(2), 561-562.

Buko, V. U., Lukivskaya, O. Y., Naruta, E. E., \& Belonovskaya, E. B. (2014). Horst-dietmar tauschel, protective effects of norursodeoxycholic acid versus ursodeoxycholic acid on thioacetamide-induced rat liver fibrosis. Journal of Clinical and Experimental Hepatology, 4(4), 293-301.

Bundy, R., Walker, A. F., Middleton, R. W., Marakis, G., \& Booth, J. C. (2004). Artichoke leaf extract reduces symptoms of irritable bowel syndrome and improves quality of life in otherwise healthy volunteers suffering from concomitant dyspepsia: A subset analysis. Journal of Altemative and Complementary Medicine, 10(4), 667-669.

Chan, W. K., Ida, N. H., Cheah, P. L., \& Goh, K. L. (2014). Progression of liver disease in non-alcoholic fatty liver disease: A prospective clinicopathological follow-up study. Journal of Digestive Diseases, 15, 545-552.

Chen, W., \& Gluud, C. (2003). Bile acids for primary sclerosing cholangitis. Cochrane database of systematic reviews, 2, CD003626.

Chen, X. D., Zhao, S. M., Hua, X. Y., \& Yu, H. (2010). Angelica effects on proliferation and differentiation of neural stem cells from neonatal rats with intrauterin hypoxia. Journal of Clinical Rehabilitative Tissue Engineering Research, 14(27), 5050-5053.

Cheng, K., Ashby, D., \& Smyth, R. L. (2012). Ursodeoxycholic acid for cystic fibrosis-related liver disease. Cochrane Database Systematic Reviews, 10, CD000222.

Chernobrovy, V. M. (2013). Bile reflux gastritis and esophagitis: The urgent questions of treatment with Ukrliv - ursodeoxycholic acid (future trends in diagnosis using 24-hour multichannel intraluminal impedance-pH-monitoring). Modern Gastroenterology, 2, 47-53 (in Ukrainian).

Chesney, R. W. (1985). Taurine: Its biological role and clinical implications. Advances in Pediatrics, 32, 1-42.

Chhabra, S. K., Ahmed, Z., Massey, A., Agarwal, S., Vij, V., Agarwal, B., Vij, V., Agarwal, B., Kankaria, J., \& Jenaw, R. K. (2016). Laparoscopic cholecystectomy in a 2 year old male child with choleilithiasis and recurrent right hypochondrial pain: Case report and review of literature. Intemational Journal of Surgery Case Reports, 26, 142-145.

Coats, M., \& Shimi, S. M. (2015). Cholecystectomy and the risk of alimentary tract cancers: A systematic review. World Journal of Gastroenterology, 21(12), 3679-3693.

Colombo, C., Battezzati, P. M., Podda, M., Bettinardi, N., \& Giunta, A. (1996). Ursodeoxycholic acid for liver disease associated with cystic fibrosis: A doubleblind multicenter trial. The Italian Group for the Study of Ursodeoxycholic Acid in Cystic Fibrosis. Hepatology, 23(6), 1484-1490. 
Cong, W. M., Dong, H., Tan, L., Sun, X. X., \& Wu, M. C. (2011). Surgicopathological classification of hepatic space-occupying lesions: A single-center experience with literature review. World Journal of Gastroenterology: WJG, 17(19), 2372-2378.

Crosignani, A., Battezzati, P. M., Setchell, K. D., Invernizzi, P., Covini, G., Zuin, M., Podda, M. (1996). Tauroursodeoxycholic acid for treatment of primary biliary cirrhosis. A dose-response study. Digestive Diseases and Sciences, 41(4), 809-815.

De la Puerta, C., Arrieta, F. J., Balsa, J. A., Botella-Carretero, J. I., Zamarrón, I., \& Vázquez, C. (2010). Taurine and glucose metabolism: A review. Nutrición Hospitalaria, 25(6), 910-919.

De Luca, A., Pierno, S., \& Camerino, D. C. (2015). Taurine: The appeal of a safe amino acid for skeletal muscle disorders. Journal of Translational Medicine, 13, 243.

Dhami, N., Dev Mishra, A. (2015). Phytochemical variation: How to resolve the quality controversies of herbal medicinal products? Journal of Herbal Medicine, 5(2), 118-127.

Dilger, K., Hohenester, S., Winkler-Budenhofer, U., Bastiaansen, B. A., Schaap, F. G., Rust, C., \& Beuers, U. (2012). Effect of ursodeoxycholic acid on bile acid profiles and intestinal detoxification machinery in primary biliary cirrhosis and health. Journal of Hepatology, 57(1), 133-140.

Dong, W. G., Liu, S. P., Zhu, H. H., Luo, H. S., \& Yu, J. P. (2004). Abnormal function of platelets and role of angelica sinensis in patients with ulcerative colitis. World Journal of Gastroenterology, 10(4), 606-609.

Dymowski, W. (2013). Assessment report on Angelica sinensis (Oliv.) Diels, radix. EMA/HMPC/614586/2012. Committee on Herbal Medicinal Products, European Medicines Agency, United Kingdom, London.

Englisch, W., Beckers, C., Unkauf, M., Ruepp, M., \& Zinserling, V. (2000). Efficacy of artichoke dry extract in patients with hyperlipoproteinemia. Arzneimittelforschung, 50(3), 260-265.

Fang, C., Filipp, F. V., \& Smith, J. W. (2012). Unusual binding of ursodeoxycholic acid to ileal bile acid binding protein: role in activation of FXR $\alpha$. Journal of Lipid Research, 53(4), 664-673.

Fang, L., Xiao, X., Liu, C., \& He, X. (2012). Recent advance in studies on Angelica sinensis. Chinese Herbal Medicines, 4(1), 12-25.

Ferracane, R., Pellegrini, N., Visconti, A., Graziani, G., Chiavaro, E., Miglio, C., \& Fogliano, V. (2008). Effects of different cooking methods on antioxidant profile, antioxidant capacity, and physical characteristics of artichoke. Journal of Agricultural and Food Chemistry, 56(18), 8601-8608.

Frolov, V. M., Garnik, T. P., \& Peresadin, M. O. (2009). Effect of phyto-preparations from artichokes of barbed on lipo peroxidation indices in patients with non-alcoholic steatohepatitis, combined with chronic noncalculous cholecystitis and obesity. Ukrainian Medical Almanac, 12(4), 185-191 (in Ukrainian)

Galkin, A. Y., \& Kotov, A. G. (2011). Development of quality control methods and analysis of herbal preparation for treatment and prevention of alopecia. Ukrainian Journal of Clinical and Laboratory Medicine, 6(2), 115-119 (in Ukrainian).

Galkin, A. Y., Solovjova, V. F., \& Dugan, A. M. (2013). Anti-inflammatory and immunomodulating properties of the herbal preparation indicated for prevention and treatment of alopecia. Botanics: Targets and Therapy, 3, 49-55.

Gan, T., Liu, Y. D., Wang, Y., \& Yang, J. (2010). Traditional chinese medicine herbs for stopping bleeding from haemorrhoids. Cochrane Database of Systematic Reviews, 10, CD006791.

Gebara, E., Udry, F., Sultan, S., \& Toni, N. (2015). Taurine increases hippocampal neurogenesis in aging mice. Stem Cell Research, 14(3), 369-379.

Gérard, P. (2014). Metabolism of cholesterol and bile acids by the gut microbiota. Pathogens, 3(1), 14-24.

Ghandforoush-Sattari, M., Mashayekhi, S., Krishna, C. V., Thompson, J. P., \& Routledge, P. A. (2010). Pharmacokinetics of oral taurine in healthy volunteers. Journal of Amino Acids, 2010, ID 346237.

Giacosa, A., Guido, D., Grassi, M., Riva, A., Morazzoni, P., Bombardelli, E., Perna, S., Faliva, M. A., \& Rondanelli, M. (2015). The effect of ginger (Zingiber officinalis) and artichoke (Cynara cardunculus). Extract supplementation on functional dyspepsia: A randomised, double-blind, and placebo-controlled clinical trial. Evidence-Based Complementary and Alternative Medicine, 915087.

Gontova, T., Ilyinska, N., Golembiovska, O., \& Mashtaler, V. (2016). A study of the component composition of phenolic compounds obtained from Dahlia varieties. Der Pharma Chemica, 8(18), 455-459.

Gorchakova, N. A., Bondarenko, L. B., \& Galkin, A. J. (2017). Grounds of ursodeoxycholic acid, taurine and extractum folii artishoke combine use safety in the hepatobiliar system diseases. Phytotherapy, 2, 10-14 (in Ukrainian)

Grohmann, M. (1987). The activity of the neuronal and extra neuronal catecholamine-metabolizing enzymes of the perfused rat heart. Naunyn-Schmiedeberg's Archives of Pharmacology, 336, 139-147.

Gu, Y., Zhao, Y., Qian, K., \& Sun, M. (2015). Taurine attenuates hippocampal and corpus callosum damage, and enhances neurological recovery after closed head injury in rats. Neuroscience, 291, 331-340.
Guarino, M. P. L., Cocca, S., Altomare, A., Emerenziani, S., \& Cicala, M. (2013). Ursodeoxycholic acid therapy in gallbladder disease, a story not yet completed. World Journal of Gastroenterology, 19(31), 5029-5034

Guarino, M. P. L., Cong, P., Cicala, M., Alloni, R., Carotti, S., \& Behar, J. (2007) Ursodeoxycholic acid improves muscle contractility and inflammation in symptomatic gallbladders with cholesterol gallstones. Gut, 56(6), 815-820.

Guarrera, P. M., Salemo, G., \& Caneva, G. (2005). Folk phytotherapeutical plants from Maratea area (Basilicata, Italy). Journal of Ethnopharmacology, 99(3), 367-378.

Gubska, O. Y. (2013). The role and place of ursodeoxycholic acid drugs in treatment of chronic liver disease and pathology of hepatobiliary system. Liky Ukrayiny, 2, 10-13 (in Russian).

Harnois, D. M., Angulo, P., Jorgensen, R. A., Larusso, N. F., \& Lindor, K. D. (2001). High-dose ursodeoxycholic acid as a therapy for patients with primary sclerosing cholangitis. The American Journal of Gastroenterology, 96(5), 1558-1562.

Hess, L. M., Krutzsch, M. F., Guillen, J., Chow, H. H., Einspahr, J., Batta, A. K., Salen, G., Reid, M. E., Earnest, D. L., \& Alberts, D. S. (2004). Results of a phase I multiple-dose clinical study of ursodeoxycholic acid. Cancer Epidemiology, Biomarkers and Prevention, 13(5), 861-867.

Heubi, J. E., Wiechmann, D. A., Creutzinger, V., Setchell, K. D., Squires, R., Jr., Couser, R., \& Rhodes, P. (2002). Tauroursodeoxycholic acid (TUDCA) in the prevention of total parenteral nutrition-associated liver disease. The Journal of Pediatrics, 141(2), 237-242.

Hirata, J. D., Swiersz, L. M., Zell, B., Small, R., \& Ettinger, B. (1997). Does dong quai have estrogenic effects in postmenopausal women? A double-blind, placebo-controlled trial. Fertility and Sterility, 68(6), 981-986.

Hohlacheva, N. A., Sergeeva, N. N., \& Vahrushev, Y. M. (2016). Age and gender peculiarities of development of gallstone disease. Archive of Internal Medicine, 6(1), 34-39 (in Russian).

Hopf, C., Grieshaber, R., Hartmann, H., Hinrichsen, H., Eisold, M., Cordes, H. J. Greinwald, R., \& Rust, C. (2013). Therapeutic equivalence of ursodeoxycholic acid tablets and ursodeoxycholic acid capsules for the treatment of primary biliary cirrhosis. Clinical Pharmacology in Drug Development, 2, 231-236.

Hua, Y., Xue, W., Zhang, M., Wei, Y., \& Ji, P. (2014). Metabonomics study on the hepatoprotective effect of polysaccharides from different preparations of Angelica sinensis. Journal of Ethnopharmacology, 151(3), 1090-1099.

Huo, X., Juergens, S., Zhang, X., Rezaei, D., Yu, C., Strauch, E. D., Wang, J. Y., Cheng, E., Meyer, F., Wang, D. H., Zhang, Q., Spechler, S. J., \& Souza R. F. (2011). Deoxycholic acid causes DNA damage while inducing apoptotic resistance through NF- $\mathrm{BB}$ activation in benign Barrett's epithelial cells. American Journal of Physiology - Gastrointestinal and Liver Physiology, 301(2), G278-G286.

Hwang, S. J., Chan, C. Y., Lee, S. D., Wu, J. C., Tsay, S. H., \& Lo, K. J. (1993). Ursodeoxycholic acid in the treatment of primary biliary cirrhosis: A shortterm, randomized, double-blind controlled, cross-over study with long-term follow up. Journal of Gastroenterology and Hepatology, 8, 217-223.

Jacobsen, J. G., \& Smith, L. H. (1968). Biochemistry and physiology of taurine and taurine derivatives. Physiological Reviews, 48(2), 424-511.

Jin, Y., Tang, Y. P., Zhu, Z. H., Shang, E. X., Pang, H. Q., Shi, X. Q., Chen, Y. Y., Wang, J., Chang, X., Kang, A., Zhou, G. S., Shi, Y. J., Sun, J., Tang, Z. S., Li, S. P., \& Duan, J. A. (2017). Pharmacokinetic comparison of seven major bio-active components in normal and blood stasis rats after oral administration of herb pair danggui-honghua by UPLC-TQ/MS. Molecules, 22, 1746.

Jin, Y., Wu, L., Tang, Y. P., Cao, Y. J., Li, S. J., Shen, J., Yue, S. J., Qu, C., Shan, C. X., Cui, X. B., Zhang, L., \& Duan, J. A. (2016). UFLC-Q-TOF/MS based screening and identification of the metabolites in plasma, bile, urine and feces of normal and blood stasis rats after oral administration of hydroxysafflor yellow A. Journal of Chromatography B, 1012-1013, 124-129.

Keiding, S., Høckerstedt, K., Bjøro, K., Bondesen, S., Hjortrup, A., Isoniemi, H., Erichsen, C., Söderdahl, G., \& Ericzon, B. G. (1997). The nordic multicenter double-blind randomized controlled trial of prophylactic ursodeoxycholic acid in liver transplant patients. Transplantation, 63(11), 1591-1594.

Kim, H., Lee, K., Lee, K. W., Yi, N. J., Lee, H. W., Hong, G., Choi, Y., You, T., Suh, S. W., Jang, J. J., \& Suh, K. S. (2014). Histologically proven non-alcoholic fatty liver disease and clinically related factors in recipients after liver transplantation. Clinical Transplantation, 28(5), 521-529.

Kirchhoff, R., Beckers, C., Kirchhoff, G. M., Trinczek-Gäıtner, H., Petrowicz, O., \& Reimann, H. J. (1994). Increase in choleresis by means of artichoke extract. Phytomedicine, 1(2), 107-115.

Ko, W. C. (1980). A newly isolated antispasmodic - butylidenephthalide. The Japanese Journal of Pharmacology, 30(1), 85-91.

Kotb, M. A. (2012). Molecular mechanisms of ursodeoxycholic acid toxicity and side effects: Ursodeoxycholic acid freezes regeneration and induces hibernation mode. International Journal of Molecular Sciences, 13(7), 8882-8914.

Kraft, K. (1997). Artichoke leaf extract - Recent findings reflecting effects on lipid metabolism, liver and gastrointestinal tracts. Phytomedicine, 4(4), 369-378. 
Lafay, S., Morand, C., Manach, C., Besson, C., \& Scalbert, A. (2006). Absorption and metabolism of caffeic acid and chlorogenic acid in the small intestine of rats. British Journal of Nutrition, 96, 39-46.

Lafay, S., Morand, C., Manach, C., Besson, C., \& Scalbert, A. (2006). Absorption and metabolism of caffeic acid and chlorogenic acid in the small intestine of rats. British Journal of Nutrition, 96(1), 39-46.

Lammert, F., Neubrand, M. W., Bittner, R., Feussner, H., Greiner, L., Hagenmüller, F., Kiehne, K. H., Ludwig, K., Neuhaus, H., Paumgartner, G., Riemann, J. F., \& Sauerbruch, T. (2007). S-3 guidelines for diagnosis and treatment of gallstones. German Society for Digestive and Metabolic Diseases and German Society for Surgery of the Alimentary Tract. Zeitschrift Fur Gastroenterologie, 45(9), 971-1001.

Larghi, A., Crosignani, A., Battezzati, P. M., De Valle, G., Allocca, M., Invernizzi, P., Zuin, M., \& Podda, M. (1997). Ursodeoxycholic and tauro-ursodeoxycholic acids for the treatment of primary biliary cirrhosis: A pilot crossover study. Alimentary Pharmacology and Therapeutics, 11, 409-414.

Lazaridis, K. N., Gores, G. J., \& Lindor, K. D. (2001). Ursodeoxycholic acid mechanisms of action and clinical use in hepatobiliary disorders. Journal of Hepatology, 35(1), 134-146.

Li, W., Guo, J., Tang, Y., Wang, H., Huang, M., Qian, D., \& Duan, J. A. (2012). Pharmacokinetic comparison of ferulic acid in normal and blood deficiency rats after oral administration of Angelica sinensis, Ligusticum chuanxiong and their combination. International Journal of Molecular Sciences, 13(3), 3583-3597.

Lo, A. C. T., Chan, K., Yeung, J. H. K., \& Woo, K. S. (1995). Danggui (Angelica sinensis) affects the pharmacodynamics but not the pharmacokinetics of warfarin in rabbits. European Journal of Drug Metabolism and Pharmacokinetics, 20(1), 55-60.

Loseva, N. V., \& Moiseenko, E. E. (2010). Experience in the use of Dibicor in the complex therapy of non-alcoholic fatty liver disease. Pharmateca, 13, 63-67 (in Russian).

Luo, H., Xiao, F., \& Gu, F. (2003). Study on pharmacokinetic of ferulic acid and peoniflorin in Angelic-Paeonia root powder. Zhong yao cai Journal of Chinese Medicinal Materials, 23, 189-192 (in Chinese)

Lupattelli, G., Marchesi, S., Lombardini, R., Roscini, A. R., Trinca, F., Gemelli, F., Vaudo, G., \& Mannarino, E. (2004). Artichoke juice improves endothelial function in hyperlipemia. Life Sciences, 76(7), 775-782.

Lutsenko, T. N., Kovalenko, M. V., \& Galkin, O. Y. (2017). Validation of biological activity testing procedure of recombinant human interleukin-7. Ukrainian Biochemical Journal, 89(1), 82-89.

Ma, J. P., Guo, Z. B., Jin, L., \& Li, Y. D. (2015). Phytochemical progress made in investigations of Angelica sinensis (Oliv.) Diels. Chinese Journal of Natural Medicines, 13(4), 241-249.

Marcinkiewicz, J., \& Kontny, E. (2014). Taurine and inflammatory diseases. Amino Acids, 46(1), 7-20.

May, G. R., Sutherland, L. R., \& Shaffer, E. A. (1993). Efficacy of bile acid therapy for gallstone dissolution: A meta-analysis of randomized trials. Alimentary Pharmacology and Therapeutics, 7, 139-148.

Mayev, I. V., Kucheryavy, Y. A., Morozov, S. V., Stukova, N. Y. (2010). Effect of ursodeoxycholic acid drugs on blood biochemical parameters and results of liver elastography in patients with alcohol-induced liver cirrhosis. Clinical Perspectives in Gastroenterology and Hepatology, 4, 43-48 (in Russian).

Miyazaki, T., \& Matsuzaki, Y. (2014). Taurine and liver diseases: A focus on the heterogeneous protective properties of taurine. Journal of Amino Acids, 46(1), 101-110.

Mizushima, S., Nara, Y., Sawamura, M., Yamori, Y. (1996). Effects of oral taurine supplementation on lipids and sympathetic nerve tone. In: Huxtable, R. J., Azuma, J., Kuriyama, K., Nakagawa, M., \& Baba, A. (Eds.). Taurine 2. Advances in Experimental Medicine and Biology, Vol. 403. Springer, Boston.

Mootoosamy, A., \& Fawzi Mahomoodally, M. (2014). Ethnomedicinal application of native remedies used against diabetes and related complications in Mauritius. Journal of Ethnopharmacology, 151(1), 413-444.

Moridani, M. Y., Scobie, H., Jamshidzadeh, A., Salehi, P., \& O’Brien, P. J. (2001) Caffeic acid, chlorogenic acid, and dihydrocaffeic acid metabolism: Glutathione conjugate formation. Drug Metabolism and Disposition, 29(11), 1432-1439.

Naismith, D. J., Rana, S. K., \& Emery, P. W. (1987). Metabolism of taurine during reproduction in women. Human Nutrition. Clinical Nutrition, 41(1), 37-45.

Özkardeş, A. B., Tokaç, M., Dumlu, E. G., Bozkurt, B., Çiftçi, A. B., Yetişir, F., \& Kılıç, M. (2014). Early versus delayed laparoscopic cholecystectomy for acute cholecystitis: A prospective, randomized Study. International Surgery, 99(1), 56-61.

Pallayova, M., \& Taheri, S. (2014). Non-alcoholic fatty liver disease in obese adults: Clinical aspects and current management strategies. Clinical Obesity, 4, 243-253.

Pei, K., Ou, J., Huang, J., \& Ou, S. (2016). p-Coumaric acid and its conjugates: Dietary sources, pharmacokinetic properties and biological activities. Journal of the Science of Food and Agriculture, 96, 2952-2962.
Pelletier, G., Roulot, D., Davion, T., Masliah, C., Causse, X., Oberti, F., Raabe, J. J., Van Lemmens, C., Labadie, H., \& Serfaty, L. (2003). A randomized controlled trial of ursodeoxycholic acid in patients with alcohol-induced cirrhosis and jaundice. Hepatology, 37, 887-892.

Podda, M., Ghezzi, C., Battezzati, P. M., Bertolini, E., Crosignani, A., Petroni, M. L., \& Zuin, M. (1989). Effect of different doses of ursodeoxycholic acid in chronic liver disease. Digestive Diseases and Sciences, 34(12), 59S-65S.

Portincasa, P., Ciaula, A. D., Bonfrate, L., \& Wang, D. Q. (2012). Therapy of gallstone disease: What it was, what it is, what it will be. World Journal of Gastrointestinal Pharmacology and Therapeutics, 3(2), 7-20.

Ramaekers, J. G. (2017). Drugs and driving research in medicinal drug development. Trends in Pharmacological Sciences, 38(4), 319-321.

Raphael, B. P., \& Duggan, C. (2012). Prevention and treatment of intestinal failureassociated liver disease in children. Seminars Liver Disease, 32(4), 341-347.

Roma, M. G., Toledo, F. D., Boaglio, A. C., Basiglio, C. L., Crocenzi, F. A., \& Sánchez Pozzi, E. J. (2011). Ursodeoxycholic acid in cholestasis: Linking action mechanisms to therapeutic applications. Clinical Science (Lond), 121(12), 523-544.

Rost, D., Rudolph, G., Kloeters-Plachky, P., \& Stiehl, A. (2004). Effect of highdose ursodeoxycholic acid on its biliary enrichment in primary sclerosing cholangitis. Hepatology, 40, 693-698.

Ru, Y., Ge, L., Ko, N. L., \& Tam, Y. K. (2007). Low oral bioavailability and pharmacokinetics of senkyunolide A, a major bioactive component in Rhizoma Chuanxiong in the rat. Therapeutic Drug Monitoring, 29, 49-56.

Rudic, J. S., Poropat, G., Krstic, M. N., Bjelakovic, G., \& Gluud, C. (2012). Ursodeoxycholic acid for primary biliary cirrhosis. Cochrane Database of Systematic Reviews, 12, CD000551.

Sagara, M., Murakami, S., Mizushima, S., Liu, L., Mori, M., Ikeda, K., \& Yamori, Y. (2015). Taurine in 24-h urine samples is inversely related to cardiovascular risks of middle aged subjects in 50 populations of the world. Advances in Experimental Medicine and Biology, 803, 623-636.

Sahebkar, A., Pirro, M., Banach, M., Mikhailidis, D. P., Atkin, S. L., \& Cicero, A. F. G. (2017). Lipid-lowering activity of artichoke extracts: A systematic review and meta-analysis. Critical Reviews in Food Science and Nutrition, 1-8.

Sannia, A. (2010). Phytotherapy with a mixture of dry extracts with hepato-protective effects containing artichoke leaves in the management of functional dyspepsia symptoms. Minerva Gastroenterologica e Dietologica, 56(2), 93-99 (in Italian)

Santoveña, A Sánchez-Negrín, E Charola, L. Llabrés, M. \& Fariña, J. B. (2014). Study of quality and stability of ursodeoxycholic acid formulations for oral pediatric administration. Intemational Joumal of Pharmaceutics, 477(1-2), 32-38.

Sheybak, V. M., \& Sheybak, L. N. (2005). The biological role of taurine in mammals. Medical News, 10, 1-8 (in Russian).

Simoni, P., Cerrè, C., Cipolla, A., Polimeni, C., Pistillo, A., Ceschel, G., Roda, E., \& Roda, A. (1995). Bioavailability study of a new, sinking, enteric-coated ursodeoxycholic acid formulation. Pharmacological Research, 31(2), 115-119.

Sital, R. R., Kusters, J. G., De Rooij, F. W., Kuipers, E. J., \& Siersema, P. D. (2006) Bile acids and Barrett's oesophagus: A sine qua non or coincidence? Scandinavian Journal of Gastroenterology. Supplement, 243, 11-17.

Sturman, J. A., Hepner, G. W., Hofmann, A. F., \& Thomas, P. J. (1975). Metabolism of [35S]taurine in man. Journal of Nutrition, 105(9), 1206-1214.

Su, Y. W., Chiou, W. F., Chao, S. H., Lee, M. H., Chen, C. C., \& Tsai, Y. C. (2011). Ligustilide prevents LPS-induced iNOS expression in RAW 264.7 macrophages by preventing ROS production and down-regulating the MAPK, NF-кB and AP-1 signaling pathways. International Immunopharmacology, 11(9), 1166-1172.

Sumizu, K. (1962). Oxidation of hypotaurine in rat liver. Biochimica et Biophysica Acta, 63, 210-212

Thao, T. D., Ryu, H. C., Yoo, S. H., \& Rhee, D. K. (2008). Antibacterial and antiatrophic effects of a highly soluble, acid stable UDCA formula in Helicobacter pylori-induced gastritis. Biochemical Pharmacology, 75(11), 2135-2146.

Tian, Y., Yang, Z. F., Li, Y., Qiao, Y., Yang, J., Jia, Y. Y., \& Wen, A. D. (2010). Pharmacokinetic comparisons of hydroxysafflower yellow A in normal and blood stasis syndrome rats. Journal of Ethnopharmacology, 129, 1-4.

Tiedemann, F., \& Gmelin, L. (1827). Einige neue Bestandtheile der Galle des Ochsen. Annalen der Physik, 85(2), 326-337.

Tomeno, W., Kawashima, K., Yoneda, M., Saito, S., Ogawa, Y., Honda, Y., Kessoku, T., Imajo, K., Mawatari, H., Fujita, K., Saito, S., Hirayasu, Y., \& Nakajima, A. (2015). Non-alcoholic fatty liver disease comorbid with major depressive disorder: The pathological features and poor therapeutic efficacy. Journal of Gastroenterology and Hepatology, 30, 1009-1014.

Tomida, S., Abei, M., Yamaguchi, T., Matsuzaki, Y., Shoda, J., Tanaka, N., \& Osuga, T. (1999). Long-term ursodeoxycholic acid therapy is associated with reduced risk of biliary pain and acute cholecystitis in patients with gallbladder stones: A cohort analysis. Hepatology, 30, 6-13.

Toyoda, A., Koike, H., Nishihata, K., Iio, W., \& Goto, T. (2015). Effects of chronic taurine administration on gene expression, protein translation and phos- 
phorylation in the rat hippocampus. Advances in Experimental Medicine and Biology, 803, 473-480.

Triantos, C. K., Koukias, N. M., Nikolopoulou, V. N., \& Burroughs, A. K. (2011) Meta-analysis: Ursodeoxycholic acid for primary sclerosing cholangitis. Alimentary Pharmacology and Therapeutics, 34, 901-910.

Tsai, M. C., Chen, C. H., Lee, H. C., Lin, H. C., \& Lee, C. Z. (2015). Increased risk of depressive disorder following cholecystectomy for gallstones. PLoS ONE, 10(6), e0129962. http://doi.org/10.1371/journal.pone.0129962

Uvarova, K. G., Saprykina, M. M., Ahmed, M. A., Oyebamire, I. B., Makienko, N. V., Maltseva, M. S., Kamenskaya, E. P., \& Yabluchanskiy, M. I. (2014). Polymorbidity in clinical practice. The Journal of V. N. Karazin Kharkiv National University. Series: Medicine, 1141(28), 48-51.

van Stijn, M. F., Vermeulen, M. A., Siroen, M. P., Wong, L. N., van den Tol, M. P., Ligthart-Melis, G. C., Houdijk, A. P., \& van Leeuwen, P. A. (2012) Human taurine metabolism: Fluxes and fractional extraction rates of the gut, liver, and kidneys. Metabolism, 61(7), 1036-1040.

Venneman, N. G., Besselink, M. G. H., Keulemans, Y. C. A., van Berge-Henegouwen, G. P., Boermeester, M. A., Broeders, I. A. M. J., Go, P. M. N. Y. H., \& van Erpecum, K. J. (2006). Ursodeoxycholic acid exerts no beneficial effect in patients with symptomatic gallstones awaiting cholecystectomy. Hepatology, 43, 1276-1283.

Vettorazzi, J. F., Kurauti, M. A., Soares, G. M., Borck, P. C., Ferreira, S. M. Branco, R. C. S., de Souza Lima Michelone, L., Boschero, A. C., Costa Junior, J. V., \& Carneiro, E. M. (2017). Bile acid TUDCA improves insulin clearance by increasing the expression of insulin-degrading enzyme in the liver of obese mice. Scientific Reports, 7, 14876.

Wang, D. Q. H., Cohen, D. E., \& Carey, M. C. (2009). Biliary lipids and cholesterol gallstone disease. Journal of Lipid Research, 50(Suppl), S406-S411.

Wang, J., Du, J. R., Wang, Y., Kuang, X., \& Wang, C. Y. (2010). Z-ligustilide attenuates lipopolysaccharide-induced proinflammatory response via inhibiting NF- $\kappa$ B pathway in primary rat microglia. Acta Pharmacologica Sinica, 31(7), 791-797.

Wei, W. L., Zeng, R., Gu, C. M., Qu, Y., \& Huang, L. F. (2016). Angelica sinensis in China-A review of botanical profile, ethnopharmacology, phytochemistry and chemical analysis. Journal of Ethnopharmacology, 190, 116-141.

Wider, B., Pittler, M. H., Thompson-Coon, J., \& Emst, E. (2009). Artichoke leaf extract for treating hypercholesterolaemia. Cochrane Database of Systematic Reviews, 4, CD003335.
Wisher, D. (2012). Martindale: The complete drug reference. 37th ed. Journal of the Medical Library Association, 100(1), 75-76.

Wittemer, S. M., Ploch, M., Windeck, T., Müller, S. C., Drewelow, B., Derendorf, H., \& Veit, M. (2005). Bioavailability and pharmacokinetics of caffeoylquinic acids and flavonoids after oral administration of Artichoke leaf extracts in humans. Phytomedicine, 12(1-2), 28-38.

Wu, S. D., Li, L., \& Wang, J. Y. (2012). Ursodeoxycholic acid for nonalcoholic steatohepatitis. European Journal of Gastroenterology and Hepatology, 24(11), 1247-1253.

Wu, X., Qian, Z., Zhu, L., Du, F., Yung, W., Gong, Q., \& Ke, Y. (2011). Neuroprotective effect of ligustilide against ischaemia-reperfusion injury via upregulation of erythropoietin and down-regulation of RTP801. British Journal of Pharmacology, 164(2), 332-343.

Wu, Y. C., \& Hsieh, C. L. (2011). Pharmacological effects of radix Angelica sinensis (Danggui) on cerebral infarction. Chinese Medicine, 6, 32.

Yan, R., Ko, N. L., Li, S. L., Tam, Y. K., \& Lin, G. (2007). Pharmacokinetics and metabolism of ligustilide, a major bioactive component in Rhizoma Chuanxiong, in the rat. Drug Metabolism and Disposition, 36(2), 400-408.

Zaretskiy, M. M., Chemikova, N. M., \& Lobachevskaya, T. V. (2011). Possibilities of using ursodeoxycholic acid in the treatment of cholelithiasis. Modem Gastroenterology, 2, 136-140 (in Russian).

Zeng, H. T., Xue, P., Su, S. L., Huang, X. C., Shang, E. X., Guo, J. M., Qian, D. W., Tang, Y. P., \& Duan, J. A. (2016). Comparative pharmacokinetics of three major bioactive components in rats after oral administration of Typhae Pollen-Trogopterus Feces drug pair before and after compatibility. DARU Journal of Pharmaceutical Sciences, 20, 2.

Zhang, M., Bi, L. F., Fang, J. H., Su, X. L., Da, G. L., Kuwamori, T., \& Kagamimori, S. (2004). Beneficial effects of taurine on serum lipids in overweight or obese non-diabetic subjects. Amino Acids, 26(3), 267-271.

Zhao, Z., \& Moghadasian, M. H. (2010). Bioavailability of hydroxycinnamates: A brief review of in vivo and in vitro studies. Phytochemistry Reviews, 9(1), 133-145.

Zvyagintseva, T. D., \& Gridnyova, S. V. (2014). The mechanisms of development and approaches to the treatment of sphincter of oddi dysfunction after cholecystectomy. Gastroenterology, 2, 77-81 (in Russian). 